\title{
ZEROES OF QUATERNIONIC MODULAR FORMS AND CENTRAL $L$-VALUES
}

\author{
KIMBALL MARTIN AND JORDAN WIEBE
}

\begin{abstract}
Values of quaternionic modular forms are related to twisted central $L$ values via periods and a theorem of Waldspurger. In particular, certain twisted $L$ values must be non-vanishing for forms with no zeroes. Here we study, theoretically and computationally, zeroes of definite quaternionic modular forms of trivial weight. Local sign conditions force certain forms to have trivial zeroes, but we conjecture that almost all forms have no nontrivial zeroes. In particular, almost all forms with appropriate local signs should have no zeroes. We show these conjectures follow from a conjecture on the average number of Galois orbits, and give applications to (non)vanishing of $L$-values.
\end{abstract}

\section{INTRODUCTION}

1.1. Motivation. Let $S_{k}(N)$ denote the space of weight $k$ elliptic cusp forms of level $\Gamma_{0}(N)$, and let $S_{k}^{\text {new }}(N)$ denote the subspace of newforms. Let $B=B_{N}$ a definite quaternion algebra of discriminant $N$, and $\mathcal{O}=\mathcal{O}_{N}$ a maximal order of $B$. Necessarily, $N$ is a squarefree product of an odd number of primes. Let $\operatorname{Cl}(\mathcal{O})$ be the set of right $\mathcal{O}$-ideal classes in $B$, which is a finite set.

The space of trivial weight modular forms of level $\mathcal{O}$ on $B$ is simply the space of functions $M(\mathcal{O})=\{\varphi: \mathrm{Cl}(\mathcal{O}) \rightarrow \mathbb{C}\}$. The subspace of cusp forms $S(\mathcal{O})$ can be defined as the codimension 1 subspace of forms orthogonal to the constant functions, with respect to the Petersson inner product. There is a well-known action of Hecke operators, and the Eichler-Shimizu-Jacquet-Langlands correspondence gives a Hecke isomorphism of $S(\mathcal{O})$ with $S_{2}^{\text {new }}(N)$. In particular, there is a 1-1 correspondence of Hecke eigenforms $\varphi \in S(\mathcal{O})$ (modulo scalars) with newforms $f \in S_{2}(N)$.

A formula of Waldspurger [Wal85] relates certain twisted $L$-values to certain periods on $B$. Namely, if $\varphi$ and $f$ correspond as above, central $L$-values associated to certain twists of $f$ are nonzero if and only if certain linear combinations of values of $\varphi$ called periods are nonzero. Thus a natural approach to studying the vanishing of $L$-values is to study both (i) values of quaternionic modular (eigen)forms $\varphi$, and (ii) periods on $B$. While periods have often been used to study $L$-values (e.g., see [JC01], [MV07]), we understand relatively little about the values of quaternionic eigenforms.

The present paper represents one step in an attempt to understand (i) and (ii), but especially values of quaternionic modular forms. Specifically, we focus on the question of how often values of quaternionic eigenforms are zero, and what this implies about nonvanishing $L$-values. In particular, let $K=\mathbb{Q}(\sqrt{-D})$ be the imaginary quadratic field of discriminant $-D$ and $\eta_{D}(n)=\left(\frac{-D}{n}\right)$ the associated quadratic Dirichlet character. We

Date: June 19, 2020. 
show that if $\varphi$ is zero-free and either (1) $K$ has class number 1 with $\eta_{D}(p) \neq 1$ for all $p \mid N$, or (2) $K$ has one class per genus and $D \mid N$, then $L\left(\frac{1}{2}, f\right) L\left(\frac{1}{2}, f \otimes \eta_{D}\right) \neq 0$ (Theorem 1.6). Moreover, we conjecture that $\varphi$ is zero-free $100 \%$ of the time that certain necessary conditions on the Atkin-Lehner signs $w_{p}$ of $f$ are satisfied-e.g., if $w_{p}=-1$ for all $p \mid N$ (see Conjecture 1.1). Assuming these sign conditions hold, Theorem 4.4 implies that $\varphi$ is zero-free if the Galois conjugates of $f$ span the Atkin-Lehner eigenspace of $S_{2}^{\text {new }}(N)$ for the signs $w_{p}$-something that was conjectured to hold $100 \%$ of the time in [Marb]. While we do not have other simple criteria to guarantee that $\varphi$ is zero-free, it is something that one can check computationally.

We note that theorems which guarantee the nonvanishing of specific central $L$-values typically rely on special arithmetic information like the properties of certain elliptic curves or the existence of an Eisenstein series congruent to $f$. Our emphasis is that knowledge of the values of quaternionic modular forms offers a different method to obtain nonvanishing of explicit twists. This idea was also exploited in [Mar17], which used information on values of quaternionic modular forms to construct Eisenstein congruences, and then apply this to $L$-values. In fact, if $f$ satisfies Eisenstein congruence, then under some conditions one can conclude $\varphi$ is zero-free (see [Mar17, Remark 2.4]).

Next we describe the contents in more detail. We remark that much of what we say extends to Eichler and even more general orders. However, for simplicity we restrict to maximal orders for the introduction and the bulk of the paper.

1.2. Zeroes of quaternionic modular forms. First, we note that $S(\mathcal{O})$ breaks up into Hecke-invariant subspaces which are the analogue of Atkin-Lehner eigenspaces for $S_{2}^{\text {new }}(N)$. Namely, for each $p \mid N, T_{p}$ acts as an involution on $S(\mathcal{O})$, so each eigenform $\varphi \in S(\mathcal{O})$ has a collection of local signs $\varepsilon_{p}(\varphi)= \pm 1$ for $p \mid N$, which are the $T_{p}$-eigenvalues of $\varphi$. These signs are the opposite of the Atkin-Lehner signs $w_{p}$.

In [Mar18b], we observed that the local signs of $\varphi$ force $\varphi$ to be 0 on certain ideal classes. We call such zeroes trivial zeroes, and study them in Section 3. In particular, if the product of all local signs is -1 , which corresponds to $\varphi$ having root number -1 , then one necessarily has trivial zeroes. We estimate the number of trivial zeroes, and give a precise formula in the case of prime level. If all signs are +1 , then there are no trivial zeroes. If $N$ is prime, so that there is only one local sign, we thus have a complete answer to when and how many trivial zeroes there are for any eigenform. When $N$ is not prime, the situation is more complicated but we give criteria for sign patterns to induce trivial zeroes. See Proposition 3.7 for necessary and sufficient criteria when $N$ is odd.

Using a combination of numerics and heuristics, we make the following conjectures. Let $\mathrm{Sq}_{r}$ denote the positive squarefree integers with exactly $r \geq 1$ prime factors, and $\mathrm{Sq}_{r}(X)=\left\{N \in \mathrm{Sq}_{r}: N<X\right\}$. For each $N \in \mathrm{Sq}_{\text {odd }}:=\bigcup_{r \text { odd }} \mathrm{Sq}_{r}$, we choose a maximal order $\mathcal{O}_{N}$ in the definite quaternion algebra $B_{N}$ of discriminant $N$. For each $N$, there are a finite number of choices for $\mathcal{O}_{N}$ up to isomorphism, but this choice does not affect the values of the eigenforms in $S\left(\mathcal{O}_{N}\right)$ (see Lemma 2.2).

Conjecture 1.1. Fix $r$ odd. Consider the collection of all eigenforms (modulo scalars) which lie in some $S\left(\mathcal{O}_{N}\right)$ with $N \in \mathrm{Sq}_{r}$, partially ordered by level. Then we have:

(i) $100 \%$ of the zeroes of these eigenforms are trivial zeroes; and

(ii) $100 \%$ of these eigenforms have no trivial zeroes. 
Note $r=1$ here corresponds to restricting to prime levels. One can also formulate a version without fixing $r$, i.e., for $N \in \mathrm{Sq}_{\text {odd }}$.

For a given eigenform $\varphi$, its Hecke eigenvalues generate a number field $K_{\varphi}$, which equals the rationality field $K_{f}$ of the associated newform $f \in S_{2}(N)$, i.e., the number field generated by the Fourier coefficients of $f$. Define the (rationality) degree of the $\varphi$ (and also $f$ ) to be the degree $\left[K_{\varphi}: \mathbb{Q}\right]=\left[K_{f}: \mathbb{Q}\right]$ of the rationality field.

Conjecture 1.2 (Rough form). Quaternionic eigenforms are more likely to have nontrivial zeroes if they have smaller degree.

See Conjectures 5.2 and 5.3, and Remark 5.4 for more precise forms of Conjecture 1.1(i) and Conjecture 1.2. Our heuristics also lead us to briefly consider the distribution of values of quaternionic eigenforms in Section 5.3.

As evidence for Conjecture 1.2, we show that eigenforms with large degree cannot have too many zeroes (Corollary 4.3). The idea of the proof is very simple -it uses the fact that $\varphi$ and its Galois conjugates have zeroes at the same locations, and they span a $d$ dimensional subspace of $S\left(\mathcal{O}_{N}\right)$. Then one uses the description of the subspaces of $S\left(\mathcal{O}_{N}\right)$ with fixed local signs. This result also provides evidence for Conjecture 1.1, under some hypotheses about the sizes of Galois orbits. Namely, in [Marb], the first author made the following conjecture.

Conjecture 1.3. Fix $k \in 2 \mathbb{Z}_{>0}$ and $r \in \mathbb{Z}_{>0}$. The average number of Galois orbits of $S_{k}^{\text {new }}(N)$ for $N \in \mathrm{Sq}_{r}$ is $2^{r}$, i.e.,

$$
\lim _{X \rightarrow \infty} \frac{\sum_{N \in \mathrm{Sq}_{r}(X)} \# \mathrm{Orb}\left(S_{k}^{\text {new }}(N)\right)}{\# \mathrm{Sq}_{r}(X)}=2^{r} .
$$

Here \# $\operatorname{Orb}\left(S_{k}^{\text {new }}(N)\right)$ denotes the number of Galois orbits of newforms in $S_{k}(N)$.

Then the following is a simple consequence of Theorem 4.4.

Theorem 1.4. Conjecture 1.3 (for $k=2$ ) implies Conjecture 1.1(ii).

1.3. Nonvanishing of $L$-values. A well-known theorem of Waldspurger [Wal85] relates twisted central $L$-values to periods on quaternion algebras. In special situations, we can use the nonvanishing of values of quaternionic modular forms to deduce nonvanishing of certain periods, and thus of certain $L$-values.

Let $K=\mathbb{Q}(\sqrt{-D})$ be the imaginary quadratic field of discriminant $-D$. Denote by $\mathrm{Cl}(K)$ its ideal class group, $\eta_{D}(n)=\left(\frac{-D}{n}\right)$, and $\varphi_{K}$ the base change of an eigenform $\varphi$ to $K$. For a quaternionic or elliptic modular eigenform $\varphi$ and a character $\chi$ defined over the same field, let $L(s, \varphi \otimes \chi)$ denote the twisted automorphic $L$-function normalized so that $s=\frac{1}{2}$ is the central point. In particular, if $\chi$ is the trivial character of $\mathrm{Cl}(K)$, then $L\left(s, \varphi_{K} \otimes \chi\right)=L\left(s, \varphi_{K}\right)=L(s, \varphi) L\left(s, \varphi \otimes \eta_{D}\right)$.

Let $B=B_{N}$. Then $K$ embeds in $B$ if and only if $\eta_{D}(p) \neq 1$ for each $p \mid N$. We may choose a maximal order $\mathcal{O}_{N}$ of $B$ and an embedding of $K$ into $B$ such that $\mathfrak{o}_{K}$, the ring of integers of $K$, embeds into in $\mathcal{O}_{N}$. This leads to an ideal class map of sets: $\mathrm{Cl}(K) \rightarrow \mathrm{Cl}\left(\mathcal{O}_{N}\right)$. We study properties of this map in Section 6.1.

Now for a character $\chi$ of $\mathrm{Cl}(K)$ and $\varphi \in S\left(\mathcal{O}_{N}\right)$, we define a period $P_{K, \chi}(\varphi)$ which is a sum of $h_{K}$ (not necessarily distinct) values of $\varphi$ in terms of this ideal class map. Assuming $\varphi$ is an eigenform, [Wal85] tells us that $P_{K, \chi}(\varphi) \neq 0$ implies $L\left(\frac{1}{2}, \varphi_{K} \otimes \chi\right) \neq 0$. Under certain local conditions the converse is also true. 
We say an eigenform $\varphi \in S(\mathcal{O})$ is zero-free if $\varphi(x) \neq 0$ for all $x \in \operatorname{Cl}(\mathcal{O})$. From Conjecture 1.1, we expect that $100 \%$ of eigenforms $\varphi$ with appropriate local sign conditions are zero-free. It is immediate that if $h_{K}=1$ and $\varphi$ is zero-free, then $P_{K, 1}(\varphi) \neq 0$. More generally, orthogonality of characters implies the following.

Theorem 1.5 (see Theorem 6.12). Suppose $N \in \mathrm{Sq}_{\mathrm{odd}}$, and let $K$ be a quadratic field embedding in $B_{N}$. Assume $\varphi \in S\left(\mathcal{O}_{N}\right)$ is zero-free. Then there exists a character $\chi$ of $\mathrm{Cl}(K)$ such that $L\left(\frac{1}{2}, \varphi_{K} \otimes \chi\right) \neq 0$.

We note that for any $\varphi$ with root number +1 , the conclusion follows for $K$ of sufficiently large discriminant via equidistribution of the ideal class maps (see [Mic04], [MV07]). However, our emphasis is that the above is valid for any $K$ embedding in $B_{N}$, which lets us conclude nonvanishing for specific $K$.

The most interesting case is when $\chi=1$, as then one can conclude both $L\left(\frac{1}{2}, \varphi\right) \neq 0$ and the quadratic Dirichlet twist $L\left(\frac{1}{2}, \varphi \otimes \eta_{D}\right) \neq 0$. Using properties of the ideal class map, we can show the following. Let us call an imaginary quadratic $K=\mathbb{Q}(\sqrt{-D})$ special for $B$ if either (i) $h_{K}=1$ and $K$ embeds in $B$, or (ii) $K$ has one class per genus (i.e., the class group has exponent 2) and $p \mid D$ implies $p \mid N$. (The latter condition implies $K$ embeds in $B$.) E.g., the class number 2 field $K=\mathbb{Q}(\sqrt{-5})$ is special for any $B_{N}$ such that $10 \mid N$.

Theorem 1.6 (see Theorem 6.10). Suppose $N \in \mathrm{Sq}_{\mathrm{odd}}$ and $\varphi \in S\left(\mathcal{O}_{N}\right)$ is zero-free. Then $L\left(\frac{1}{2}, \varphi_{K}\right) \neq 0$ for all $K$ which are special for $B_{N}$.

While there is no apparent way of expressing the condition that $\varphi \in S\left(\mathcal{O}_{N}\right)$ is zero-free directly in terms of the associated newform $f \in S_{2}(N)$, we briefly explain two ways one can computationally verify this from the perspective of classical modular forms. First, suppose $p \nmid N$ is such that $T_{p}$ acts on $S_{2}^{\text {new }}(N)$ with distinct Hecke eigenvalues. One can realize $T_{p}$ as a Brandt matrix, and $\varphi$ as an eigenvector of this matrix for the eigevalue $a_{p}(f)$. So $\varphi$ being zero-free means that an eigenvector of a Brandt matrix has no zero entries. This is the direct, computational approached we used to generate our data.

Here is another situation in which one can check $\varphi$ is zero-free using some of our results below. Suppose the Atkin-Lehner eigenvalues $w_{p}(f)=-1$ for all $p \mid N$, or that the dimension of the minimal Atkin-Lehner eigenspace containing $f$ is one more than the dimension of the Atkin-Lehner eigenspace with all Atkin-Lehner eigenvalues $w_{p}=-1$. If the Galois conjugates of $f$ generate the whole Atkin-Lehner eigenspace, then $\varphi$ is zerofree by Theorem 4.4 and the condition in (3.2). In either of these situations, Theorem 1.6 tell us that $L\left(\frac{1}{2}, f\right) L\left(\frac{1}{2}, f \otimes \eta_{K}\right) \neq 0$ for any special $K$ embedding in $B$.

Note that there are only finitely many $K$ which are special for a given $B$. In fact, there are at most 66 one-class-per-genus imaginary quadratic fields [Wei73], so the collection of $K$ to which Theorem 1.6 applies is rather small. However, this still may be of some interest as there are very few general results about nonvanishing of specific twists of specific forms which do not use special arithmetic constructions such as Eisenstein congruences. This is somewhat general in the sense that, for any given one-class-per-genus $K$, there are infinitely many $B$ for which $K$ is special. The analytic rank of $\varphi$ is defined to be the order of vanishing of $L(s, \varphi)$ at $s=\frac{1}{2}$. If $B$ is such that there exists a special $K$ for $B$ (which holds for more than $99 \%$ of prime discriminant $B$ ), then $\varphi$ being zero-free is a sufficient condition for having analytic rank 0 (i.e., $L\left(\frac{1}{2}, \varphi\right) \neq 0$ ). 
Being more speculative, we can view the proportion of values of an eigenform $\varphi \in$ $S\left(\mathcal{O}_{N}\right)$ which are 0 as a proxy for the probability that a special base change $\varphi_{K}$ has analytic rank $\geq 2$, assuming each local sign is +1 (see Proposition 6.8). (This proxy is not meant to be taken too literally, but experimentally rank 2 forms tend to have more zeroes than rank 0 forms.) Thus the proportion of values of $\varphi$ which are nonzero is a proxy for an upper bound on the probability that $\varphi$ has analytic rank $\geq 2$. This combined with Conjecture 1.2 (or rather the more precise Conjecture 5.3), and the proliferance of rank 2 elliptic curves, suggests that most high rank newforms come from elliptic curves:

Conjecture 1.7. Among all weight 2 newforms with analytic rank $\geq 2,100 \%$ have degree 1 -i.e., rational Fourier coefficients - when partially ordered by level.

To elaborate a little more on our reasoning for Conjecture 1.7, from [Marb] we expect that for $N$ squarefree the set of newforms in $S_{2}^{\text {new }}(N)$ with fixed Atkin-Lehner signs $w_{p}$ for all $p \mid N$ should decompose into 1 large Galois orbit and at most a few small Galois orbits. As $N \rightarrow \infty$, it is conjectured that $50 \%$ of newforms in $S_{2}^{\text {new }}(N)$ have analytic rank 0 and $50 \%$ have analytic rank 1 (e.g., see [ILS00]). Consequently, one - and therefore every - form in a large Galois orbit should have analytic rank $\leq 1$, i.e., all newforms with analytic rank $\geq 2$ should have small degree.

Now [Marb] suggests most small degree newforms are in fact degree 1. Further, Conjecture 5.3 suggests that the probability, as a function of $N$, that a degree $d$ form has rank $\geq 2$ tends to 0 notably faster for $d>1$ than for $d=1$. These expectations lead to Conjecture 1.7 .

We remark that, according to data in the LMFDB, for weight 2 newforms of level $\Gamma_{0}(N)$ with $N<10000$ (not necessarily in Sqodd or squarefree), there are 2436 Galois orbits with analytic rank at least 2. (In fact, only one has rank bigger than 2, corresponding to the rank 3 elliptic curve of conductor 5077.) Of these 1970 are degree 1, 372 are degree 2, 74 are degree 3,18 are degree 4, and 2 are degree 5 . These data, while not ample, agree with the above expectations.

Acknowledgements. We thank David Farmer for useful comments. We also appreciate the detailed and thoughtful feedback of the referee. The first author was supported by a grant from the Simons Foundation/SFARI (512927, KM).

Notation. Throughout $B$ denotes a definite quaternion algebra over $\mathbb{Q}$ and $\mathcal{O}$ an order in $B$. Initially $\mathcal{O}$ is allowed to be an Eichler or sometimes more general order. However, from Section 3.2 onwards we assume $\mathcal{O}$ is maximal. Denote by $\operatorname{Cl}(\mathcal{O})=\left\{x_{1}, \ldots, x_{h}\right\}$ the set of invertible right $\mathcal{O}$-ideal classes in $B$, and $h=|\mathrm{Cl}(\mathcal{O})|$ the class number of $\mathcal{O}$.

Let $\operatorname{Ram}(B)$ be the set of finite rational primes ramified in $B$. Let $\Delta=\prod_{p \in \operatorname{Ram}(B)} p$ be the discriminant of $B$, and $N$ the level of $\mathcal{O}$. Note that some authors normalize levels so that the level of a maximal order is 1 , and some so that the level of a maximal order is $\Delta$-we use the latter convention.

For us, $p$ always denotes a finite rational prime. For a $\mathbb{Z}$-module $M$, we denote by $M_{p}=M \otimes_{\mathbb{Z}} \mathbb{Z}_{p}$ the localization at $p$. Set $\hat{\mathcal{O}}^{\times}=\prod \mathcal{O}_{p}^{\times}$and $\hat{B}=\prod^{\prime} B_{p}^{\times}$, where $\prod^{\prime}$ means the restricted direct product with respect to $\left\{\mathcal{O}_{p}^{\times}\right\}$.

Similarly, if $K$ is a number field, we let $\mathfrak{o}_{K}$ be its integer ring, $\mathrm{Cl}(K)$ its class group, $h_{K}$ its class number, and let $\hat{\mathfrak{o}}_{K}^{\times}$and $\hat{K}^{\times}$denote the usual restricted products of the localized 
multiplicative groups over finite primes. In Section $6, K=\mathbb{Q}(\sqrt{-D})$ will always denote an imaginary quadratic field of discriminant $-D$.

For $N \in \mathbb{Z}_{>0}$, denote by $\omega(N)$ the number of prime divisors of $N$.

With apologies to the reader, $\varepsilon$ is used to denote a sign or a collection thereof, and $\epsilon$ a positive real number.

\section{QuATERNIONIC MODUlar FORMS}

Here we review the theory of definite quaternionic modular forms of trivial weight and make some basic observations. Some references for quaternionic modular forms and their connection to classical modular forms are [DV13], [Mar17] and [Mar]. For simplicity, we work over $\mathbb{Q}$, and primarily with Eichler orders, but the theory extends to totally real number fields and more general orders.

Let $B / \mathbb{Q}$ be a definite quaternion algebra of discriminant $\Delta$, and $\mathcal{O}$ an order in $B$. We identify the set $\mathrm{Cl}(\mathcal{O})$ of invertible right $\mathcal{O}$-ideal classes with $B^{\times} \backslash \hat{B}^{\times} / \hat{\mathcal{O}}^{\times}$. Here $B^{\times}$is diagonally embedded in $\hat{B}^{\times}$. For ease of notation, for a function $f$ on $\mathrm{Cl}(\mathcal{O})$, we write $f(x)$ for $f\left(B^{\times} x \hat{\mathcal{O}}^{\times}\right)$where $x \in \hat{B}^{\times}$.

We define the space of quaternionic modular forms on $B$ of level $\mathcal{O}$ (with trivial weight and trivial central character) to be

$$
M(\mathcal{O})=\{\varphi: \operatorname{Cl}(\mathcal{O}) \rightarrow \mathbb{C}\} .
$$

Let $x_{1}, \ldots, x_{h} \in \hat{B}^{\times}$be a set of representatives for $\mathrm{Cl}(\mathcal{O})$, and fix right $\mathcal{O}$-ideal class representatives $\mathcal{I}_{i}$ corresponding to each $x_{i}$. Let $e_{i}=\left[\mathcal{O}_{l}\left(\mathcal{I}_{i}\right)^{\times}: \mathbb{Z}^{\times}\right]$, where $\mathcal{O}_{l}\left(\mathcal{I}_{i}\right)$ denotes the left order of $\mathcal{I}_{i}$, i.e., $\mathcal{O}_{l}\left(\mathcal{I}_{i}\right)=x_{i} \hat{\mathcal{O}} x_{i}^{-1} \cap B$.

Consider the Petersson inner product on $M(\mathcal{O})$ given by

$$
\left(\varphi, \varphi^{\prime}\right)=\sum_{i=1}^{h} \frac{1}{e_{i}} \varphi\left(x_{i}\right) \overline{\varphi^{\prime}\left(x_{i}\right)} .
$$

For $\alpha \in \hat{B}^{\times}$, define the Hecke operator

$$
T_{\alpha}(\varphi)(x)=\sum \varphi(x \beta)
$$

where $\beta$ runs over a set of representatives for $\hat{\mathcal{O}}^{\times} \alpha \hat{\mathcal{O}}^{\times} / \hat{\mathcal{O}}^{\times}$so that $\hat{\mathcal{O}}^{\times} \alpha \hat{\mathcal{O}}^{\times}=\bigsqcup \beta \hat{\mathcal{O}}^{\times}$. For a positive integer $n$, define $T_{n}=\sum T_{\alpha}$ where $\alpha$ runs over a set of elements in $\hat{B}^{\times}$ such that $\bigsqcup \hat{\mathcal{O}}^{\times} \alpha \hat{\mathcal{O}}^{\times}=\left\{x \in \hat{\mathcal{O}}:\left|N_{B / \mathbb{Q}}(x)\right|=n\right\}$. Here $N_{B / \mathbb{Q}}$ denotes the reduced norm from $B$ to $\mathbb{Q}$. Classically, we may view $T_{n} \varphi$ as the sum of right translates of $\varphi$ by the integral right $\mathcal{O}$-ideals of norm $n$. The Hecke operators $T_{n}$ are a commuting family of self-adjoint operators with respect to $(\cdot, \cdot)$, which are algebraically generated by the $T_{p}$ 's.

For simplicity, we now assume $\mathcal{O}$ is Eichler or more generally special of unramified quadratic type in the terminology of [Mar]. Let $N$ be the level of $\mathcal{O}$. Then the class number $h$ of $\mathcal{O}$ only depends on $\Delta$ and $N$. See [HPS89] for a formula for $h$. We call the Hecke algebra generated over $\mathbb{Z}$ by all $T_{p}$ 'sacting on $M(\mathcal{O})$ or $S(\mathcal{O})$ the full Hecke algebra. The subalgebra generated by all $T_{p}$ 's with $p \nmid N$ is called the unramified or spherical Hecke algebra.

Let $\mathbb{1} \in M(\mathcal{O})$ denote the constant function 1 . Then $\mathbb{C} \mathbb{1}$ is a Hecke-invariant subspace of $M(\mathcal{O})$ (for $p \nmid N$, a local calculation shows there are $p+1$ right $\mathcal{O}$-ideals of norm $p$, so $\left.T_{p} \mathbb{1}=(p+1) \mathbb{1}\right)$, which we think of as the Eisenstein subspace of $M(\mathcal{O})$. We define the 
space $S(\mathcal{O})$ of cusp forms to be the orthogonal complement of $\mathbb{1}$ in $M(\mathcal{O})$, i.e., $\varphi \in S(\mathcal{O})$ if and only if $\sum \frac{1}{e_{i}} \varphi\left(x_{i}\right)=0$. By an eigenform of $M(\mathcal{O})$, we mean an element $\varphi$ of $M(\mathcal{O})$ which is a simultaneous eigenvector of all $T_{p}$ 's for $p \nmid N$. If $\mathcal{O}$ is maximal, this is equivalent to saying that $\varphi$ is a simultaneous eigenvector for every $T_{p}$ (or every $T_{n}$ ).

The (Eichler-Shimizu-)Jacquet-Langlands correspondence induces a monomorphism of Hecke modules from $S(\mathcal{O}) \rightarrow S_{2}(N)$ for the unramified Hecke algebra, and one can describe the image as a Hecke module as in [Mar]. In the special case that $\mathcal{O}$ is maximal, we in fact get Hecke module isomorphisms with newspaces for the full Hecke algebra:

$$
M(\mathcal{O}) \simeq \mathbb{C} E_{2, N} \oplus S_{2}^{\text {new }}(N), \quad S(\mathcal{O}) \simeq S_{2}^{\text {new }}(N),
$$

Here $E_{2, N}$ is an Eisenstein series in $M_{2}(N)$ with the appropriate ramified Hecke eigenvalues (see [Mar17] or [Mar]). For $N$ prime, we may take $E_{2, N}$ the unique Eisenstein series in $M_{2}(N)$ with first Fourier coefficient 1.

Example 2.1. Let $N=37, B$ be the quaternion algebra of discriminant $N$, and $\mathcal{O}$ be a maximal order in $B$. For prime discriminants $N$, one can use the Eichler mass formula and class number formula to determine $h$ and the $e_{i}$ 's in terms of the congruence class of $N \bmod 12$. E.g., see [Gro87, Table 1.3]. In this case, the class number is $h=3$ and each $e_{i}=1$. Hence $S(\mathcal{O})=\left\{\varphi \in M(\mathcal{O}): \sum_{i=1}^{3} \varphi\left(x_{i}\right)=0\right\}$.

We compute (the Brandt matrix for) the Hecke operator $T_{2}$ in Sage with the command BrandtModule(37).hecke_matrix (2). This gives $T_{2}=\left(\begin{array}{lll}1 & 1 & 1 \\ 1 & 0 & 2 \\ 1 & 2 & 0\end{array}\right)$. Since $T_{2}$ acts on $\mathbb{C} E_{2, N} \oplus S_{2}^{\text {new }}(N)$, and thus $M(\mathcal{O})$, with distinct eigenvalues, it generates the Hecke algebra over $\mathbb{C}$, and $\varphi$ in $M(\mathcal{O})$ will be an eigenform if and only if $\left(\varphi\left(x_{1}\right), \varphi\left(x_{2}\right), \varphi\left(x_{3}\right)\right)$ is an eigenvector of $T_{2}$. Hence a basis of eigenforms for $M(\mathcal{O})$ is given by the following table:

\begin{tabular}{c|ccc|c} 
& $x_{1}$ & $x_{2}$ & $x_{3}$ & $\varepsilon_{37}$ \\
\hline $\mathbb{1}$ & 1 & 1 & 1 & +1 \\
$\varphi_{1}$ & 2 & -1 & -1 & +1 \\
$\varphi_{2}$ & 0 & 1 & -1 & -1
\end{tabular}

The $x_{i}$ column represents the value $\varphi\left(x_{i}\right)$, and the $\varepsilon_{37}$ column represents the eigenvalue for $T_{N}=T_{37}$, which for $N$ prime gives the root number of the eigenform.

Note $S_{2}(37)$ contains two newforms $f_{1}, f_{2}$. These are both rational (degree 1 ) and thus correspond to the two isogeny classes of elliptic curves of conductor 37. Say $f_{1}$ has root number +1 and $f_{2}$ has root number -1 . Then the Jacquet-Langlands correspondence says that, for all Hecke operators $T_{n}, \mathbb{1}$ has the same Hecke eigenvalues as $E_{2,37}$, and $\varphi_{i}$ has the same Hecke eigenvalues as $f_{i}$ for $i=1,2$.

The following lemma is valid for arbitrary orders.

Lemma 2.2. If $\mathcal{O}$ and $\mathcal{O}^{\prime}$ are locally isomorphic orders in $B$ (i.e., $\mathcal{O}_{p} \simeq \mathcal{O}_{p}^{\prime}$ for all $p)$, then there is a bijection $\iota: \operatorname{Cl}\left(\mathcal{O}^{\prime}\right) \rightarrow \mathrm{Cl}(\mathcal{O})$ such that $\varphi \mapsto \varphi \circ \iota$ defines a Hecke isomorphism $\iota^{*}: M(\mathcal{O}) \rightarrow M\left(\mathcal{O}^{\prime}\right)$ for the full Hecke algebra, i.e., $\iota^{*}\left(T_{n} \varphi\right)=T_{n}\left(\iota^{*} \varphi\right)$ for all $n$.

Proof. Since $\mathcal{O}$ and $\mathcal{O}^{\prime}$ are locally isomorphic, there exists $\xi \in \hat{B}^{\times}$such that $\hat{\mathcal{O}}^{\prime}=\xi \hat{\mathcal{O}} \xi^{-1}$. The map $x \mapsto x \xi$ for $x \in \hat{B}^{\times}$induces a bijection $\iota: \mathrm{Cl}\left(\mathcal{O}^{\prime}\right) \rightarrow \mathrm{Cl}(\mathcal{O})$. 
If we let $\gamma \in \hat{\mathcal{O}} / \hat{\mathcal{O}}^{\times}$run over the integral right $\mathcal{O}$-ideals of norm $n$, then

$$
\iota^{*}\left(T_{n} \varphi\right)(x)=\left(T_{n} \varphi\right)(x \xi)=\sum \varphi(x \xi \gamma)=\sum \varphi\left(x \gamma^{\prime} \xi\right)=T_{n}\left(\iota^{*} \varphi\right)(x),
$$

where $\gamma^{\prime}=\xi \gamma \xi^{-1}$, as then $\gamma^{\prime}$ runs over the integral right $\mathcal{O}^{\prime}$-ideals of norm $n$.

The genus of $\mathcal{O}$ is the collection of all orders $\mathcal{O}^{\prime}$ in $B$ which are locally isomorphic to $\mathcal{O}$. The number of isomorphism classes of orders in the genus of $\mathcal{O}$ is finite, and is called the type number of $\mathcal{O}$.

Consequently, with the notation as in the lemma, if we take a basis of eigenforms $\left\{\varphi_{i}\right\}$ for $M(\mathcal{O})$, then $\left\{\varphi_{i} \circ \iota\right\}$ is a basis of eigenforms for $M\left(\mathcal{O}^{\prime}\right)$. If $\mathcal{O}$ is Eichler (or special of unramified quadratic type), then the genus of $\mathcal{O}$ simply consists of all Eichler (or special of unramified quadratic type) orders $\mathcal{O}^{\prime} \subset B$ of level $N$ [HPS89]. Now Lemma 2.2 tells us that the sets of values of the eigenforms in $M(\mathcal{O})$ only depend upon the genus of $\mathcal{O}$, which for our class of orders only depends upon $\Delta$ and $N$.

Suppose now $\mathcal{O}$ is maximal (so $N=\Delta$ ). Then, given any two eigenforms $\varphi, \varphi^{\prime} \in M(\mathcal{O})$, their collections of Hecke eigenvalues agree for all $T_{n}$ (or even almost all $T_{p}$ ) if and only if $\varphi$ and $\varphi^{\prime}$ are scalar multiples. Thus the multiset of values $\left\{\varphi\left(x_{1}\right), \ldots, \varphi\left(x_{h}\right)\right\}$ of an eigenform $\varphi$ for a maximal order $\mathcal{O}$ is, up to a scalar multiple, determined by its Hecke eigenvalues. In particular, the set of zeroes of $\varphi$ is determined its Hecke eigenvalues. Moreover, the number of zeroes of such a $\varphi$ is an invariant of the associated newform $f \in S_{2}(N)$, i.e., it does not depend upon the choice of $\mathcal{O}$ or the choice of $\varphi$ (provided $\Delta=N)$.

In Section 6, we also consider quaternionic modular forms from the representationtheoretic perspective. Trivial weight automorphic forms for $B^{\times}\left(\mathbb{A}_{\mathbb{Q}}\right)$ with trivial character are simply functions $\varphi: B^{\times} \mathbb{A}_{\mathbb{Q}}^{\times} \backslash B^{\times}\left(\mathbb{A}_{\mathbb{Q}}\right) / B^{\times}(\mathbb{R}) \simeq B^{\times} \hat{\mathbb{Q}}^{\times} \backslash \hat{B}^{\times} \rightarrow \mathbb{C}$. Via the right regular representation, this space of automorphic forms decomposes into a direct sum of irreducible automorphic representations $\pi$, and correspondingly $M(\mathcal{O})$ breaks up as a direct sum of $\hat{\mathcal{O}}^{\times}$-invariant functions: $M(\mathcal{O})=\bigoplus \pi^{\hat{\mathcal{O}}^{\times}}$. Similarly, $S(\mathcal{O})=\bigoplus \pi^{\hat{\mathcal{O}}^{\times}}$, where $\pi$ runs over the irreducible automorphic representations of $B^{\times}\left(\mathbb{A}_{\mathbb{Q}}\right)$ which are not 1 dimensional (so necessarily infinite dimensional).

\section{LOCAL INVOLUTIONS AND TRIVIAL ZEROES}

In this section, we study the zeroes of quaternionic modular forms forced by local sign conditions, which we call trivial zeroes. Recall $\Delta$ is the discriminant of $B$. We assume now $\mathcal{O} \subset B$ is Eichler of level $N$. From Section 3.2 onwards, we will further assume $\mathcal{O}$ is maximal, i.e., $N=\Delta$.

3.1. Local involutions and trivial zeroes. Consider a prime $p \mid N$. If $p \mid \Delta$, then $\mathcal{O}_{p}$ is the unique maximal order in $B_{p}$, and we let $\varpi_{p}=\varpi_{B_{p}}$ denote a uniformizer in $\mathcal{O}_{p}$. If $p \nmid \Delta$, then $\mathcal{O}_{p} \subset B_{p}=M_{2}\left(\mathbb{Q}_{p}\right)$ is a local Eichler order of some level $p^{e}$, and we can choose $\varpi_{p}=\varpi_{\mathcal{O}_{p}} \in \mathcal{O}_{p}$ such that $\varpi_{p}$ is $\mathrm{GL}_{2}\left(\mathbb{Q}_{p}\right)$-conjugate to $\left(p^{e}{ }^{1}\right)$ and $\varpi_{p}$ normalizes $\mathcal{O}_{p}$.

In either case, we lift our local $\varpi_{p} \in \mathcal{O}_{p}$ to the element $\hat{\varpi}_{p}=\left(1, \ldots, 1, \varpi_{p}, 1, \ldots\right) \in \hat{B}^{\times}$, which normalizes $\hat{\mathcal{O}}^{\times}$. We may assume $\varpi_{p}^{2}=p^{v_{p}(N)} \in Z\left(B_{p}^{\times}\right) \simeq \mathbb{Q}_{p}^{\times}$. Thus right multiplication by $\hat{\varpi}_{B_{p}}$ gives an action on $\mathrm{Cl}(\mathcal{O})$ which is a permutation of order at most 
2. Denote this involution by $\sigma_{p}$, which we will also view as the corresponding permutation of our fixed set of representatives $\left\{x_{1}, \ldots, x_{h}\right\}$.

We can also view this action classically in terms of ideals. Let $\operatorname{Pic}(\mathcal{O})$ denote the Picard group of 2-sided invertible $\mathcal{O}$-ideals modulo $\mathbb{Q}^{\times}$. Each $\hat{\varpi}_{B_{p}}$ corresponds to a 2sided (prime) $\mathcal{O}$-ideal $\mathfrak{P}_{p}$ of norm $p$, and these ideals form a set of generators for $\operatorname{Pic}(\mathcal{O}) \simeq$ $(\mathbb{Z} / 2 \mathbb{Z})^{\omega(N)}$. Then $\operatorname{Pic}(\mathcal{O})$ acts on $\mathrm{Cl}(\mathcal{O})$ by right multiplication, with multiplication by the class of the $\mathfrak{P}_{p}$ acting by $\sigma_{p}$.

If $p \mid \Delta$, the Hecke operator $T_{\hat{\varpi}_{p}}=T_{p}$, with $T_{p}$ defined as above. However, if $p \mid N$ but $p \nmid \Delta$, then $T_{\hat{\varpi}_{p}}$ is not the $T_{p}$ defined in the previous section, but is the analogue of the Atkin-Lehner operator $W_{p}$ on $M(\mathcal{O})$. E.g., if $p \| N$ but $p \nmid \Delta$, then $T_{\hat{\varpi}_{p}}$ acts as $-T_{p}$ on $S(\mathcal{O})$ and as the identity on $\mathbb{C} \mathbb{1}$.

Now $\varphi \in M(\mathcal{O})$ being an eigenform for $T_{\hat{\varpi}_{p}}$ for some $p \mid N$ with eigenvalue $\varepsilon_{p}= \pm 1$ is equivalent to the statement that

$$
\varphi\left(\sigma_{p}\left(x_{i}\right)\right)=\varepsilon_{p} \varphi\left(x_{i}\right), \quad 1 \leq i \leq h .
$$

In particular, if $x_{i}$ is a fixed point of the involution $\sigma_{p}$, then any $\varphi$ in the $(-1)$-eigenspace for $T_{\hat{\varpi}_{p}}$ must necessarily vanish on $x_{i}$. For instance, in Example 2.1, one can deduce from (3.1) that $\sigma_{37}$ permutes $x_{2}$ and $x_{3}$ and fixes $x_{1}$, which forces $\varphi_{2}\left(x_{1}\right)=0$. This is the simplest way in which the local involutions $\sigma_{p}$ can force an eigenform $\varphi$ to have zeroes, but there are others.

For instance, suppose $p$ and $q$ are primes dividing $\Delta$, and $\sigma_{p}$ and $\sigma_{q}$ both interchange $x_{1}$ and $x_{2}$. If the $T_{p}$ and $T_{q}$ eigenvalues of $\varphi$ have opposite signs, then we are forced to have $\varphi\left(x_{1}\right)=\varphi\left(x_{2}\right)=0$ (as in Example 3.2 below). Alternatively, suppose $p, q$ and $r$ are primes dividing $\Delta$, and $\sigma_{p}$ (resp. $\sigma_{q}$, resp. $\sigma_{r}$ ) interchanges $x_{1}$ and $x_{2}$ (resp. $x_{2}$ and $x_{3}$, resp. $x_{3}$ and $x_{1}$ ). Then if $\varphi$ is a form in the $(-1)$-eigenspace for $T_{p}$ and the (+1)-eigenspaces for $T_{q}$ and $T_{r}$, we must have

$$
\varphi\left(x_{1}\right)=-\varphi\left(x_{2}\right)=-\varphi\left(x_{3}\right)=-\varphi\left(x_{1}\right),
$$

forcing $\varphi\left(x_{1}\right)$ (and thus $\varphi\left(x_{2}\right)=\varphi\left(x_{3}\right)$ ) to be zero.

Let $X_{1}, \ldots, X_{t}$ denote the set of orbits of $\mathrm{Cl}(\mathcal{O})$ under the action of $\operatorname{Pic}(\mathcal{O})$. We remark that two ideal classes in $\mathrm{Cl}(\mathcal{O})$ have isomorphic left orders if and only if they lie in the same orbit under $\operatorname{Pic}(\mathcal{O})$. Consequently, $t$ is the type number of $\mathcal{O}$.

By a sign pattern $\varepsilon$ for $N$, we mean a multiplicative function $d \mapsto \varepsilon_{d}$ from the set of divisors $d$ of $N$ to $\{ \pm 1\}$. Consider the associated eigenspace for $\left(T_{\hat{\varpi}_{p}}\right)_{p \mid N}$,

$$
M^{\varepsilon}(\mathcal{O})=\left\{\varphi \in M(\mathcal{O}): T_{\hat{\varpi}_{p}}(\varphi)=\varepsilon(p) \varphi \text { for } p \mid N\right\} .
$$

Now, as in [Mar18b], we define a signed (multi)graph $\Sigma^{\varepsilon}$ on $\mathrm{Cl}(\mathcal{O})$ as follows. Begin with an empty graph on $\mathrm{Cl}(\mathcal{O})$. For $p \mid N$, we adjoin an edge between $x_{i}$ and $\sigma_{p}\left(x_{i}\right)$ with sign $\varepsilon_{p}$ for each orbit $\left\{x_{i}, \sigma_{p}\left(x_{i}\right)\right\}$ of $\sigma_{p}$. (If $x_{i}$ is a fixed point of $\sigma_{p}$, we are adjoining a signed loop.) Call the resulting graph $\Sigma^{\varepsilon}$. Note that its connected components are $X_{1}, \ldots, X_{t}$.

For a (not necessarily simple) path in $\Sigma^{\varepsilon}$, define its parity to be the product of the signs of its edges. By a cycle we will simply mean a closed path, (allowing for loops as well as repeated vertices and edges). We call a component $X_{j} \varepsilon$-admissible if there are no cycles of parity -1 in $\Sigma^{\varepsilon}$ that are supported on $X_{j}$.

Consider any $\varphi \in M^{\varepsilon}(\mathcal{O})$. If $x, x^{\prime} \in X_{j}$, then by definition there is a sequence $p_{1}, \ldots, p_{m}$ of (not necessarily distinct) primes dividing $N$ such that $\left(\sigma_{p_{m}} \circ \cdots \circ \sigma_{p_{1}}\right)(x)=x^{\prime}$. 
Consequently, the values of $\varphi$ on all ideal classes in a given $X_{j}$ are determined by the value on a single $x \in X_{j}$, and in fact $\varphi\left(x^{\prime}\right)= \pm \varphi(x)$ for $x, x^{\prime} \in X_{j}$. Here the sign must be the parity of the path in $\Sigma^{\varepsilon}$ given by $x, \sigma_{p_{1}}(x), \ldots,\left(\sigma_{p_{m}} \circ \cdots \circ \sigma_{p_{1}}\right)(x)$. In particular, when $x^{\prime}=x$ this path is a cycle, and if it has parity -1 , then we must have $\varphi(x)=0$. We call such a zero of $\varphi$ a trivial zero, i.e., we say $\varphi(x)$ is a trivial zero if $\varphi \in M^{\varepsilon}(\mathcal{O})$ and the $\operatorname{Pic}(\mathcal{O})$-orbit of $x$ is not $\varepsilon$-admissible. Since this notion of a trivial zero only depends on $\varepsilon$ and the orbit $X$ rather than on $\varphi$ and $x$, we sometimes simply say that $x$ or $X$ is a trivial zero for $\varepsilon$.

Lemma 3.1. There exists some $\varphi \in M^{\varepsilon}(\mathcal{O})$ which is nonzero on $X_{j}$ if and only if $X_{j}$ is $\varepsilon$-admissible.

Proof. The "only if" direction was shown above. Conversely, suppose $X_{j}$ is $\varepsilon$-admissible. Define a nonzero $\varphi$ supported on $X_{j}$ as follows. Fix some $x \in X_{j}$ and set $\varphi(x)=1$. For any $x^{\prime} \in X_{j}$, choose a sequence of $p_{1}, \ldots, p_{m}$ of primes dividing $N$ which corresponds to a path from $x$ to $x^{\prime}$ as above. Put $\varphi\left(x^{\prime}\right)= \pm 1$ where \pm is the parity of this path in $\Sigma^{\varepsilon}$. Note that, by admissibility, the parity is independent of the choice of path (otherwise we could concatenate them to get a cycle of parity -1). In particular, $\varphi\left(\sigma_{p}\left(x_{i}\right)\right)=\varepsilon_{p} \varphi\left(x_{i}\right)$ for $p \mid N$ and $1 \leq i \leq h$, i.e., $\varphi \in M^{\varepsilon}(\mathcal{O})$.

Since we may take a basis for $M^{\varepsilon}(\mathcal{O})$ which consists of forms $\varphi$ that are supported on a single connected component $X_{j}$, this implies that $\operatorname{dim} M^{\varepsilon}(\mathcal{O})$ equals the number of $X_{j}$ which are $\varepsilon$-admissible. Thus the set of trivial zeroes for $\varepsilon$, i.e., the union of the $\varepsilon$-inadmissible orbits, is precisely the set of $x \in \mathrm{Cl}(\mathcal{O})$ such that $\varphi(x)=0$ for all $\varphi \in M^{\varepsilon}(\mathcal{O})$.

Example 3.2. Here is a basis of eigenforms for a maximal order $\mathcal{O}$ in the quaternion algebra $B$ of discriminant $\Delta=N=154=2 \cdot 7 \cdot 11$, for a suitable ordering of ideal classes. The $\varepsilon_{p}$ columns denote the associated eigenvalue for $T_{p}=T_{\hat{\varpi}_{p}}$, where $p \mid N$.

\begin{tabular}{c|cccccc|c|c|c} 
& $x_{1}$ & $x_{2}$ & $x_{3}$ & $x_{4}$ & $x_{5}$ & $x_{6}$ & $\varepsilon_{2}$ & $\varepsilon_{7}$ & $\varepsilon_{11}$ \\
\hline $\mathbb{1}$ & 1 & 1 & 1 & 1 & 1 & 1 & + & + & + \\
$\varphi_{1}$ & 1 & 1 & $\alpha$ & $\alpha$ & $-2 \alpha-2$ & $-2 \alpha-2$ & + & + & + \\
$\varphi_{2}$ & 1 & 1 & $\bar{\alpha}$ & $\bar{\alpha}$ & $-2 \bar{\alpha}-2$ & $-2 \bar{\alpha}-2$ & + & + & + \\
$\varphi_{3}$ & 0 & 0 & 0 & 0 & 1 & -1 & + & - & - \\
$\varphi_{4}$ & 1 & -1 & 0 & 0 & 0 & 0 & - & - & + \\
$\varphi_{5}$ & 0 & 0 & 1 & -1 & 0 & 0 & - & - & -
\end{tabular}

Here $\alpha=\frac{-3+\sqrt{5}}{2}$ and $\bar{\alpha}=\frac{-3-\sqrt{5}}{2}$. One can deduce from this table that there are 3 orbits: $X_{1}=\left\{x_{1}, x_{2}\right\}, X_{2}=\left\{x_{3}, x_{4}\right\}$ and $X_{3}=\left\{x_{5}, x_{6}\right\}$. Moreover, in cycle notation $\sigma_{2}$ acts as $\left(x_{1} x_{2}\right)\left(x_{3} x_{4}\right), \sigma_{7}$ acts as $\left(x_{1} x_{2}\right)\left(x_{3} x_{4}\right)\left(x_{5} x_{6}\right)$, and $\sigma_{11}$ acts as $\left(x_{3} x_{4}\right)\left(x_{5} x_{6}\right)$. The signed graph $\Sigma^{\varepsilon}$ is
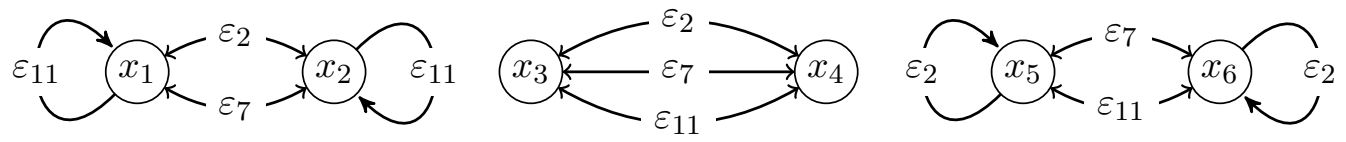

In particular, unless all the signs are the same, we have trivial zeroes on $X_{2}$-indeed, $\varphi_{3}$ and $\varphi_{4}$ are zero on $X_{2}$. If $\varepsilon_{11}=-1$ or if $\varepsilon_{2}$ and $\varepsilon_{7}$ have opposite signs (as for $\varphi_{3}$ and $\varphi_{5}$ ), we have trivial zeroes on $X_{1}$. Similarly, if $\varepsilon_{2}=-1$ or if $\varepsilon_{7}$ and $\varepsilon_{11}$ have opposite signs 
(as for $\varphi_{4}$ and $\varphi_{5}$ ), we have trivial zeroes on $X_{3}$. Hence all zeroes in the table are trivial zeroes.

Let $+_{N}\left(\right.$ resp. $\left.-{ }_{N}\right)$ denote the sign pattern for $N$ which is +1 (resp. -1$)$ for all divisors (resp. prime divisors) of $N$.

Proposition 3.3. Let $\varepsilon$ be a sign pattern for $N$. Then the number of orbits $X_{j}$ on which $\varepsilon$ has trivial zeroes is $\operatorname{dim} M^{+{ }_{N}}(\mathcal{O})-\operatorname{dim} M^{\varepsilon}(\mathcal{O})$. In particular, $\varepsilon$ has no trivial zeroes if and only if $\operatorname{dim} M^{\varepsilon}(\mathcal{O})=\operatorname{dim} M^{{ }_{N}}(\mathcal{O})$.

In particular, no eigenform in $M^{+N}(\mathcal{O})$ has trivial zeroes.

Proof. As explained above, $\operatorname{dim} M^{\varepsilon}(\mathcal{O})$ is the number of $\varepsilon$-admissible orbits $X_{j}$. Hence it suffices to show that all orbits are $+_{N}$-admissible. However, this is obvious as all cycles have parity +1 for $\Sigma_{+_{N}}$.

For example, if $N=\Delta=30$ so $\mathcal{O}$ is maximal, then $h=2$. Let $\varepsilon$ be the sign pattern with $\varepsilon_{2}=\varepsilon_{5}=-1$ and $\varepsilon_{3}=1$. Then $\operatorname{dim} M^{\varepsilon}(\mathcal{O})=\operatorname{dim} M^{+30}(\mathcal{O})=1$, so the eigenform $\varphi \in M^{\varepsilon}(\mathcal{O})$ (which corresponds to the unique newform in $S_{2}^{\text {new }}(30)$ ) has no trivial zeroes. In fact, we may scale $\varphi$ so that $\varphi\left(x_{1}\right)=-\varphi\left(x_{2}\right)=1$, and $\varphi$ has no zeroes.

3.2. Dimension formulas. From now on, we assume $\mathcal{O}$ is a maximal order in $B$, i.e., $N=\Delta$. In this situation, we use refined dimension formulas from [Mar18] to get more explicit results on trivial zeroes for sign patterns. The restriction to $\mathcal{O}$ maximal is largely for simplicity - one should be able to similarly treat Eichler orders of squarefree level with an extension of [Mar18]. In principle, one could also consider Eichler orders of nonsquarefree level, but then the relevant dimension formulas are much more complicated.

Lemma 3.4. The involution $\sigma_{N}=\prod_{p \mid N} \sigma_{p}$ acts on $\mathrm{Cl}(\mathcal{O})$ with fixed points.

Proof. By identifying $M(\mathcal{O})$ with $\mathbb{C}^{h}$ via $\varphi \mapsto\left(\varphi\left(x_{1}\right), \ldots, \varphi\left(x_{h}\right)\right)$, we see from (3.1) that we can represent $T_{N}$ by a matrix which agrees with the matrix representation for the permutation $\sigma_{N}$ of $\left\{x_{1}, \ldots, x_{h}\right\}$. In particular, $\operatorname{tr} T_{N}$ is the number of fixed points of $\sigma_{N}$.

Now

$$
\operatorname{tr} T_{N}=1+\operatorname{dim} S_{2}^{\text {new }}(N)^{+}-\operatorname{dim} S_{2}^{\text {new }}(N)^{-},
$$

where $S_{2}^{\text {new }}(N)^{ \pm}$is denotes the subspace of forms with root number \pm 1 . However, [Mar18, Corollary 2.3] tells us that $\operatorname{dim} S_{2}^{\text {new }}(N)^{+} \geq \operatorname{dim} S_{2}^{\text {new }}(N)^{-}$, so $\operatorname{tr} T_{N} \geq 1$. Hence $\sigma_{N}$ has fixed points.

Note that this lemma is really special to $\sigma_{N}$-it is not valid for a single $\sigma_{p}$ in general. E.g., $\sigma_{7}$ from Example 3.2 acts with no fixed points. More generally, see Lemma 3.9.

Corollary 3.5. If $\varepsilon$ is a sign pattern for $N$ such that $\varepsilon_{N}=-1$, then any eigenform in $M^{\varepsilon}(\mathcal{O})$ has trivial zeroes.

In other words, this says that any $\varphi$ which corresponds to a newform $f \in S_{2}(N)$ with root number -1 has trivial zeroes.

Proof. If $\varepsilon_{N}=-1$, then any fixed point of $\sigma_{N}$ must be a trivial zero for $\varepsilon$. 
In the simple case that $N$ is prime and $\mathcal{O}$ is maximal, there are only two sign patterns, $+_{N}$ and $-{ }_{N}$, and we know that all eigenforms for $+_{N}$ have no trivial zeroes and all eigenforms in $-{ }_{N}$ have trivial zeroes. When $N$ is not prime, the situation is more complicated, as we explain presently.

Denote by $a_{n}(f)$ the $n$-th Fourier coefficient of an elliptic modular form $f \in M_{2}(N)$. Let $E_{2, N}$ denote the normalized weight 2 Hecke eigenform Eisenstein series in $M_{2}(N)$ with Fourier coefficients $a_{p}\left(E_{2, N}\right)=1$ for each $p \mid N$. Put

$$
M_{2}^{\text {new }}(N)^{*}=\mathbb{C} E_{2, N} \oplus S_{2}^{\text {new }}(N) .
$$

By a newform in $M_{2}^{\text {new }}(N)^{*}$, we will mean either a newform in $S_{2}^{\text {new }}(N)$ or $E_{2, N}$. For $p \mid N$, let $W_{p}$ denote the Atkin-Lehner operator on $M_{2}^{\text {new }}(N)^{*}$, which is defined as usual on $S_{2}^{\text {new }}(N)$ and and acts by -1 on $\mathbb{C} E_{2, N}$. Then $W_{p}(f)=-a_{p}(f)$ for each newform in $M_{2}^{\text {new }}(N)^{*}$.

Let $\varepsilon$ be a sign pattern for $N$. Put

$$
M_{2}^{\text {new }, \varepsilon}(N)^{*}=\left\{f \in M_{2}^{\text {new }}(N)^{*}: W_{p} f=\varepsilon(p) f \text { for } p \mid N\right\},
$$

and similarly $S_{2}^{\text {new }, \varepsilon}(N)=M_{2}^{\text {new }, \varepsilon}(N)^{*} \cap S_{2}^{\text {new }}(N)$. Note $\operatorname{dim} M_{2}^{\text {new }, \varepsilon}(N)^{*}$ is simply $1+$ $\operatorname{dim} S_{2}^{\text {new }, \varepsilon}(N)$ if $\varepsilon=-N$ and $\operatorname{dim} S_{2}^{\text {new }, \varepsilon}(N)$ otherwise.

Denote by $-\varepsilon$ the sign pattern for $N$ which has the opposite signs as $\varepsilon$ at all primes $p \mid N$. Then the Jacquet-Langlands correspondence gives an isomorphism of full Hecke modules

$$
M^{\varepsilon}(\mathcal{O}) \simeq M_{2}^{\text {new },-\varepsilon}(N)^{*} .
$$

Hence by Proposition 3.3, a form in $M^{\varepsilon}(\mathcal{O})$ will have no trivial zeroes if and only if $\varepsilon=+_{N}$ or

$$
\operatorname{dim} S_{2}^{\text {new },-\varepsilon}(N)=1+\operatorname{dim} S_{2}^{\text {new, }-N}(N) .
$$

The instances where (3.2) holds with $\varepsilon \neq+_{N}$ and $N<100$ occur when $N \in\{30,42,70,78\}$. In these examples $\operatorname{dim} S_{2}^{\text {new }}(N)=1$ but $\operatorname{dim} S_{2}^{\text {new, }-N}(N)=0$. The unique newforms of these levels have root number +1 with 1 Atkin-Lehner sign -1 and 2 Atkin-Lehner signs +1 . The values of the associated quaternionic modular forms are given in [Wie19, Appendix A], which tabulates quaternionic eigenforms for maximal orders of levels $<100$. These forms have no zeroes, trivial or not. Here is the next non-trivial instance when (3.2) holds.

Example 3.6. Let $N=105$. Here $\operatorname{dim} S_{2}^{\text {new }}(N)=3$. We order the newforms $f_{1}, f_{2}, f_{3} \in$ $S_{2}(N)$ so that $f_{1}$ has rationality field $\mathbb{Q}$ and $f_{2}, f_{3}$ are the Galois conjugate forms with rationality field $\mathbb{Q}(\sqrt{5})$. Then $f_{1}$ has all 3 Atkin-Lehner signs -1 , and $f_{2}, f_{3}$ both have Atkin-Lehner signs $w_{3}=w_{5}=+1$ and $w_{7}=-1$.

Taking $\mathcal{O}$ to be a maximal order in the quaternion algebra $B$ of discriminant $N$, we compute (in Magma or using class number and mass formulas) that $h=4$ and each $e_{i}=1$. We may order $\mathrm{Cl}(\mathcal{O})$ so that a basis of eigenforms is given by the following table:

\begin{tabular}{c|cccc|c|c|c} 
& $x_{1}$ & $x_{2}$ & $x_{3}$ & $x_{4}$ & $\varepsilon_{3}$ & $\varepsilon_{5}$ & $\varepsilon_{7}$ \\
\hline $\mathbb{1}$ & 1 & 1 & 1 & 1 & + & + & + \\
$\varphi_{1}$ & 1 & 1 & -1 & -1 & + & + & + \\
$\varphi_{2}$ & 1 & -1 & $2-\sqrt{5}$ & $-2+\sqrt{5}$ & - & - & + \\
$\varphi_{3}$ & 1 & -1 & $2+\sqrt{5}$ & $-2-\sqrt{5}$ & - & - & +
\end{tabular}


If we assume $f_{2}, f_{3}$ are ordered so that $a_{2}\left(f_{2}\right)=\sqrt{5}$ and $a_{2}\left(f_{3}\right)=-\sqrt{5}$, then $\varphi_{i}$ corresponds to $f_{i}$ for each $1 \leq i \leq 3$. Here we see that (3.2) holds with $\varepsilon$ given by $\left(\varepsilon_{3}, \varepsilon_{5}, \varepsilon_{7}\right)=(-,-,+)$, and the eigenforms in $M^{\varepsilon}(\mathcal{O})$ have no zeroes, trivial or not.

Denote by $\Delta_{d}$ the discriminant of $\mathbb{Q}(\sqrt{-d})$ and $h\left(\Delta_{d}\right)$ its class number.

Proposition 3.7. Assume $N$ is odd, and let $\varepsilon \neq+_{N}$ be a sign pattern for $N$. Let $S$ be the set of divisors $d>1$ of $N$ such that $\left(\frac{\Delta_{d}}{p}\right)=-1$ for all $p \mid \frac{N}{d}$. Then $\varepsilon$ has no trivial zeroes if and only if both of the following conditions hold:

(i) $\varepsilon_{d}=1$ for all $d \in S$; and

(ii) if $3 \mid N$, then $\varepsilon_{3}=1$ or $N$ is divisible by some prime $p \equiv 1 \bmod 3$.

Proof. It follows from Propositions 1.4 and 3.2 of [Mar18] (or more directly from Theorem 3.3 of op. cit. if $3 \nmid N)$ that $2^{\omega(N)}\left(\operatorname{dim} M^{+N}(\mathcal{O})-\operatorname{dim} M^{\varepsilon}(\mathcal{O})\right)$ equals

(3.3) $\frac{1}{2} \sum_{1<d \mid N}\left(1-\varepsilon_{d}\right) h^{\prime}\left(\Delta_{d}\right) b(d, N / d) \prod_{p \mid \frac{N}{d}}\left(1-\left(\frac{\Delta_{d}}{p}\right)\right)+\delta_{3 \mid N} \frac{\left(1-\varepsilon_{3}\right)}{3} \prod_{p \mid \frac{N}{3}}\left(1-\left(\frac{-3}{p}\right)\right)$.

Here we use the same notation as in op. cit.: each $h^{\prime}\left(\Delta_{d}\right)$ is a weighted class number and $b(d, N / d)$ is 1,2 or 4 depending only on $d \bmod 8$. For $N$ odd, $b(d, N / d)=b(d, 1)$, where $b(d, 1)$ is given by $(3.4)$ below. In addition $\delta_{3 \mid N}$ means 1 if $3 \mid N$ and 0 otherwise. The point is that each term in the above sum is $\geq 0$, so $\operatorname{dim} M^{+N}(\mathcal{O})=\operatorname{dim} M^{\varepsilon}(\mathcal{O})$ if and only if each term is 0 . Condition (i) is equivalent to each term in the first sum being 0 , and condition (ii) is equivalent to the final term being 0 .

Remark 3.8. (a) Since these conditions are complicated, it is not obvious how frequently they are satisfied. In Sage, we computed these conditions for the 820 odd squarefree levels $N<10000$ which are products of 3 primes. ${ }^{1}$ Of these levels, 465 have at least one sign pattern $\varepsilon \neq+_{N}$ with no trivial zeroes. The total number of such sign patterns 559 . Thus it appears relatively common that for (non-prime) $N$ as above there is at least one sign pattern besides $+_{N}$ with no trivial zeroes.

(b) While we still have similar dimension formulas when $N$ is even, the issue with the above argument is that the constants $b(d, N / d)$ are $-1,-2$ or 0 if $N / d$ is even, so one cannot use a positivity argument. We note that of the 980 even levels $N \in \mathrm{Sq}_{3}$ with $N<10000,372$ have a sign pattern $\varepsilon \neq+_{N}$ with no trivial zeroes.

There is a simpler sufficient condition (in addition to the root number condition) which allows us to say certain sign patterns must have trivial zeroes. We recall the following.

Lemma 3.9. ([Mar18b, Lemma 4.3]) Let $p \mid N$. Then $\sigma_{p}$ acts on $\mathrm{Cl}(\mathcal{O})$ without fixed points if and only if

(1) $p$ is odd and $\left(\frac{-p}{q}\right)=1$ for some odd prime $q \mid N$;

(2) $p \equiv 7 \bmod 8$ and $N$ is even; or

(3) $p=2,\left(\frac{-2}{q}\right)=1$ for some prime $q \mid N$, and $N$ has a prime factor which is $1 \bmod 4$.

Thus if some $p \mid N$ does not satisfy any of these conditions, i.e., if $\sigma_{p}$ acts with fixed points, then any sign pattern $\varepsilon$ for $N$ with $\varepsilon_{p}=-1$ has trivial zeroes (and the number of trivial zeroes is at least the number of fixed points of $\sigma_{p}$ ).

\footnotetext{
${ }^{1}$ Code may be found at: https://math.ou.edu/ kmartin/data/
} 
3.3. Counting trivial zeroes. Recall that $\mathcal{O}$ is maximal.

Proposition 3.10. (i) Fix $r$ odd. As $N \rightarrow \infty$ among elements of $\mathrm{Sq}_{r}$, the maximum number of trivial zeroes for a sign pattern for $N$ is $\ll_{r} N^{\frac{1}{2}} \log N$.

(ii) Fix $\epsilon>0$. As $N \rightarrow \infty$ along $\mathrm{Sq}_{\text {odd }}$, the maximum number of trivial zeroes for a sign pattern for $N$ is $O\left(N^{\frac{1}{2}+\epsilon}\right)$.

For comparison, we note that the total number $h$ of values for a quaternionic modular form in $M(\mathcal{O})$ is asymptotic to $\frac{\varphi(N)}{12}$, where here $\varphi$ is the Euler totient function.

Proof. Let $N \in \mathrm{Sq}_{r}$ and $\varepsilon$ be a sign pattern for $N$. Recall the number of $\operatorname{Pic}(\mathcal{O})$-orbits $X_{j}$ on which $\varepsilon$ has trivial zeroes is precisely $\operatorname{dim} M^{+_{N}}(\mathcal{O})-\operatorname{dim} M^{\varepsilon}(\mathcal{O})$. Moreover, since each orbit is generated by $r$ commuting involutions, each orbit has size at most $2^{r}$. Hence for (i) it suffices to show $\operatorname{dim} M^{+_{N}}(\mathcal{O})-\operatorname{dim} M^{\varepsilon}(\mathcal{O})=O\left(N^{1 / 2} \log N\right)$. Up to the addition of a bounded term when $N$ is even, this difference of dimensions is still given by a formula of the form (3.3) (cf. [Mar18]), and thus for a suitable constant $C$ is bounded by

$$
2^{r} C \sum_{d \mid N} h\left(\Delta_{d}\right) \ll 4^{r} N^{\frac{1}{2}} \log N
$$

Here we used the standard upper bound $h\left(\Delta_{d}\right) \ll \sqrt{d} \log d \leq \sqrt{N} \log N$ and the fact that $N$ has $2^{r}$ divisors. This gives (i).

To get (ii), it suffices to show that $4^{r}=O\left(N^{\epsilon}\right)$ where $r=\omega(N)$ and $N$ is squarefree. Clearly $N \geq p_{1} \ldots p_{r}$, where $p_{i}$ is the $i$-th prime number. The growth of the primorial function $\prod_{i \leq r} p_{i}$ is known to be $e^{(1+o(1)) r \log r}$, thus $N$ grows at least as fast as $r^{r}$. From this it follows that $4^{r}=O\left(N^{\epsilon}\right)$ for any $\epsilon>0$.

Remark 3.11. In fact one can say a bit more about the sizes of orbits of $\operatorname{Pic}(\mathcal{O})$ and trivial zeroes. First each orbit has size $2^{j}$ for some $j \leq r$ (see [Mar18b, Section 4.4]). One can show that an orbit of size $2^{j}$ is inadmissible for some sign pattern $\varepsilon$ if and only if $j<r$. So one can replace $2^{r}$ by $2^{r-1}$ in the bound in the above proof. Further, from the asymptotic equidistribution of sign patterns (see op. cit.) one sees that almost all orbits must have maximal size $2^{r}$ as $N \rightarrow \infty$ along $\mathrm{Sq}_{r}$.

Let us now treat the case where $N$ is prime in more detail. In this case, there is only a single local involution $\sigma_{N}$ to consider, and we know from Lemma 3.4 (or Lemma 3.9) that $\sigma_{N}$ necessarily has fixed points. Moreover the sign pattern $+_{N}$ has no trivial zeroes, and the number of trivial zeroes of $-_{N}$ must be the number of fixed points of $\sigma_{N}$.

For $d$ odd, define

$$
b(d, 1)= \begin{cases}1 & d \equiv 1 \bmod 4 \\ 2 & d \equiv 7 \bmod 8 \\ 4 & d \equiv 3 \bmod 8\end{cases}
$$

Proposition 3.12. For $N>3$ prime, the sign pattern $-_{N}$ has exactly $\frac{1}{2} h\left(\Delta_{N}\right) b(N, 1)$ trivial zeroes.

Proof. The number of fixed points of $\sigma_{N}$ is $\operatorname{dim} M^{+_{N}}(\mathcal{O})-\operatorname{dim} M^{-N}(\mathcal{O})$. This is precisely $\frac{1}{2} h\left(\Delta_{N}\right) b(N, 1)$, which is a special case of either (3.3) or [Mar18, Theorem 2.2]. 
Note that this exact formula in the prime level case tells us that Proposition 3.10 is close to being sharp.

Corollary 3.13. For any $\epsilon>0$, as $N \rightarrow \infty$ along primes, the number of trivial zeroes for the sign pattern $-_{N}$ is $\gg N^{\frac{1}{2}-\epsilon}$.

\section{BOUNDS ON NONTRIVIAL ZEROES}

As before, $\mathcal{O} \subset B$ is a maximal order of level $N$. We first note the following trivial upper bounds on the number of zeroes of an arbitrary (not necessarily eigen) form $\varphi$.

Lemma 4.1. Let $\varphi \in S(\mathcal{O})$ be nonzero. Then $\varphi$ is nonzero on at least $\max \left\{2, \frac{(\varphi, \varphi)}{\|\varphi\|_{\infty}^{2}}\right\}$ elements of $\mathrm{Cl}(\mathcal{O})$.

Proof. Let $n_{\varphi}$ denote the number of zeroes of $\varphi$. The lower bound of $\frac{(\varphi, \varphi)}{\|\varphi\|_{\infty}^{2}}$ follows from $(\varphi, \varphi) \leq \sum\left|\varphi\left(x_{i}\right)\right|^{2} \leq\left(h-n_{\varphi}\right)\|\varphi\|_{\infty}^{2}$ and the lower bound of 2 follows from from $(\varphi, \mathbb{1})=\sum \frac{1}{e_{i}} \varphi\left(x_{i}\right)=0$.

We also note that if $\varphi \in S(\mathcal{O})$ is a nonzero eigenform, we may scale its entries to be real. Then at least one value must be strictly positive and at least one value must be strictly negative.

By sup norm bounds [BM13], for an eigenform $\varphi$ we have $\frac{(\varphi, \varphi)}{\|\varphi\|_{\infty}^{2}} \gg h^{\delta}$ for any $\delta<\frac{1}{24}$. This gives nontrivial bounds for the number of zeroes of an eigenform, but in fact we expect something much stronger is true.

4.1. Galois orbits of eigenforms. Let $\varphi \in M(\mathcal{O})$ be an eigenform, and $f \in S_{2}(N)$ the corresponding newform. For any $n \geq 1$, let $\lambda_{n}$ be the $T_{n}$-eigenvalue for $\varphi$. Let $K_{\varphi}$ be the field generated by all Hecke eigenvalues $\lambda_{n}$. By (2.1), we know each $\lambda_{n}=a_{n}(f)$, and thus $K_{\varphi}=K_{f}$ is also the rationality field of $f$.

We normalize $\varphi$ so that all of its values lie in $K_{\varphi}$. To see that this is possible, let $V$ be the space of all $K_{\varphi}$-valued functions in $M(\mathcal{O})$. Note that each $T_{n}$ acts on $V$, since each $T_{n}$ is integral (i.e., every value of $T_{n} \varphi$ is an $\mathbb{Z}$-linear combination of values of $\varphi$ ). Let $V_{0} \subset V$ be the intersection of all of the $\lambda_{n}$-eigenspaces for the $T_{n}$ 's. Note that $\operatorname{dim}_{K_{\varphi}} V_{0}=\operatorname{dim}_{\mathbb{C}} V_{0} \otimes \mathbb{C}$. The latter space is 1 -dimensional by $(2.1)$ and is spanned by $\varphi$, so some nonzero scalar multiple of $\varphi$ lies in $V_{0}$, i.e., takes values in $K_{\varphi}$. While not necessary at present, we may also scale $\varphi$ so that its values lie in the integer ring $\mathfrak{o}_{K_{\varphi}}$. We also note that $K_{\varphi}$ is the minimal number field which can contain all values of a nonzero scalar multiple of $\varphi$, as every $\lambda_{n}$ must lie must lie in the field generated by the values of $\varphi$ by the integrality of the Hecke operators.

Given an element $\tau \in \operatorname{Gal}(\mathbb{C} / \mathbb{Q})$, the Galois conjugate form $\varphi^{\tau}(x):=\tau(\varphi(x))$ is an eigenvector for each $T_{n}$ with eigenvalue $\tau\left(\lambda_{n}\right)$. Hence $\operatorname{Gal}(\mathbb{C} / \mathbb{Q})$ acts on (lines of) eigenforms, and partitions the set of (lines of) eigenforms into Galois orbits. Writing $K_{\varphi}=\mathbb{Q}(\alpha)$ for some algebraic number $\alpha$, we see the number of distinct Galois conjugates $\varphi^{\tau}$ equals the number of embeddings of $\alpha$ into $\mathbb{C}$, i.e., the degree $\left[K_{\varphi}: \mathbb{Q}\right]$ of the minimal polynomial of $\alpha$. In other words, the Galois orbit $\left\{\varphi^{\tau}: \tau \in \operatorname{Gal}(\mathbb{C} / \mathbb{Q})\right\}$ of $\varphi$ has size $\operatorname{deg}(\varphi):=\left[K_{\varphi}: \mathbb{Q}\right]$, which is the (rationality) degree of $\varphi$ (or of $f$ ). 
Clearly the zeroes of an eigenform $\varphi$ are the same as the zeroes of any conjugate $\varphi^{\tau}$. Also note that, since the full Hecke algebra is commutative, if $\varphi \in M^{\varepsilon}(\mathcal{O})$, then each conjugate $\varphi^{\tau} \in M^{\varepsilon}(\mathcal{O})$.

4.2. Fundamental domains of eigenforms. For a sign pattern $\varepsilon$ for $N$, let $\mathcal{F}_{\varepsilon}$ be a set of representatives for the $\varepsilon$-admissible orbits of $\operatorname{Pic}(\mathcal{O})$ acting on $\operatorname{Cl}(\mathcal{O})$. Thus $\# \mathcal{F}_{\varepsilon}=\operatorname{dim} M^{\varepsilon}(\mathcal{O})$, and any $\varphi \in M^{\varepsilon}(\mathcal{O})$ is determined by its values on $x \in \mathcal{F}_{\varepsilon}$. Hence we think of $\mathcal{F}_{\varepsilon}$ as a fundamental domain for functions in $M^{\varepsilon}(\mathcal{O})$.

Proposition 4.2. Suppose $\varphi \in M^{\varepsilon}(\mathcal{O})$ is a degree $d$ eigenform. Then there are at most $\# \mathcal{F}_{\varepsilon}-d$ elements $x \in \mathcal{F}_{\varepsilon}$ such that $\varphi(x)=0$.

Proof. The $d$ Galois conjugates $\varphi_{1}, \ldots, \varphi_{d}$ of $\varphi$ generate a $d$-dimensional subspace of $M^{\varepsilon}(\mathcal{O})$. On the other hand, since each $\varphi_{i}$ has the same support as $\varphi$, the dimension of the span of $\varphi_{1}, \ldots, \varphi_{d}$ is at most the number of $x \in \mathcal{F}_{\varepsilon}$ such that $\varphi(x) \neq 0$.

Since each $\varepsilon$-admissible orbit has size at most $2^{\omega(N)}$ ideal classes, we conclude the following.

Corollary 4.3. Suppose $\varphi \in M^{\varepsilon}(\mathcal{O})$ is a degree $d$ eigenform. Then $\varphi$ has at most $2^{\omega(N)}\left(\operatorname{dim} M^{\varepsilon}(\mathcal{O})-d\right)$ nontrivial zeroes.

4.3. Conditional results. Note that Conjecture 1.3 for $k=2$ and fixed $r$ implies that, as $N$ ranges over $\mathrm{Sq}_{r}, 100 \%$ of Atkin-Lehner eigenspaces $S_{2}^{\text {new, } \varepsilon}(N)$ are spanned by a single Galois orbit, and thus $100 \%$ of newforms lie in such Atkin-Lehner eigenspaces. Consequently, the following implies Theorem 1.4.

Theorem 4.4. Let $\varepsilon$ be a sign pattern for $N$. Suppose the Atkin-Lehner eigenspace $S_{2}^{\text {new, }-\varepsilon}(N)$ is spanned by a single Galois orbit. Then no eigenforms in $M^{\varepsilon}(\mathcal{O})$ have nontrivial zeroes.

Proof. If $\varepsilon \neq+_{N}$, then it is immediate from Corollary 4.3 that any of the Galois conjugate eigenforms in $M^{\varepsilon}(\mathcal{O})=S^{\varepsilon}(\mathcal{O})$ have no trivial zeroes. Also, $\mathbb{1}$ clearly has no trivial zeros.

So assume $\varepsilon=+_{N}, \operatorname{dim} S^{+_{N}}(\mathcal{O})>0$ and consider the eigenforms $\varphi_{1}, \ldots, \varphi_{d} \in$ $S^{+_{N}}(\mathcal{O})$, which are all Galois conjugate by assumption. By the same reasoning as in the proof of Proposition 4.2, each $\varphi_{i}$ must be zero-free if there exists some zero-free (not necessarily eigen) form $\varphi \in S^{+_{N}}(\mathcal{O})$.

But the existence of such a $\varphi$ is easy to see. Recall $X_{1}, \ldots, X_{t}$ are the orbits of $\mathrm{Cl}(\mathcal{O})$ under $\operatorname{Pic}(\mathcal{O})$. These are all $+_{N^{-}}$admissible, and because $\operatorname{dim} S^{+_{N}}(\mathcal{O})>0$, i.e., $\operatorname{dim} M^{+_{N}}(\mathcal{O})>1$, we have $t \geq 2$. Note $\varphi \in S^{+_{N}}(\mathcal{O})$ if and only if $\varphi$ is constant on each $X_{j}$ and $\sum_{i=1}^{h} e_{i}^{-1} \varphi\left(x_{i}\right)=0$. Put $e_{X_{j}}=e_{i}$ for any $x_{i} \in X_{j}$ - this does not depend on the choice of $x_{i}$-see [Mar18b, Lemma 4.1]. Then we may construct a zero-free $\varphi \in S^{+_{N}}(\mathcal{O})$ by choosing nonzero $a_{j}$ 's such that $\sum_{j=1}^{t} e_{X_{j}}^{-1} a_{j}=0$ and defining $\varphi=\sum a_{j} 1_{X_{j}}$.

\section{DAta AND CONJECTURES}

In this section, we present some data and conjectures on zeroes of quaternionic modular forms. Our data is based on calculations of quaternionic modular forms in both Sage [Sage] and Magma [BCP97]. ${ }^{2}$

\footnotetext{
${ }^{2}$ Some of our code is available at https://math.ou.edu/ kmartin/data/
} 
5.1. Computations. We calculate a basis of eigenforms $\varphi$ for $M(\mathcal{O})$ as follows. We use built-in functions to compute Brandt matrices $T_{p}$ acting on $M(\mathcal{O})$ for prime $N$ with Sage or general $N \in \mathrm{Sq}_{\text {odd }}$ with Magma. Given $N$, we compute $T_{p}$ for $p \nmid N$ for all small $p$ until we find a $T_{p}$ with no repeated eigenvalues. (In all cases, we find such a $p$ and it is usually the smallest $p \nmid N$.) Then the eigenvectors of $T_{p}$ give a basis of eigenforms for $M(\mathcal{O})$ as in Example 2.1, and we may scale them to make all entries algebraic integers. We compute exact eigenvectors of $T_{p}$ whenever $N<4000\left(N \in \mathrm{Sq}_{\text {odd }}\right)$ or when the degree $d$ of the (minimal polynomial of the) eigenvalue is small and $N<20000$ is prime.

Given the exact eigenvectors, we can determine the exact number and position of zeroes of our eigenforms. Then for prime level $N$, we determine which zeroes are trivial zeroes as follows. For small $N$, one can compute $T_{N}$ and determine the sign of an eigenform $\varphi$ and identify any trivial zeroes, but the calculation of $T_{N}$ is slow for $N$ large. (We expect that one should be able to implement a faster algorithm using $\sigma_{N}$, but we have not attempted this.) Instead, suppose we have computed a basis of eigenforms for $S(\mathcal{O})$. Recall for prime $N$, the trivial zeroes occur exactly at the fixed points of $\sigma_{N}$ for $\varphi \in S^{-N}(\mathcal{O})$. We know the number $r$ of fixed points of $\sigma_{N}$ from Proposition 3.12. Then we find the $x_{i}$ 's in $\mathrm{Cl}(\mathcal{O})$ such that at least $\operatorname{dim} S^{-N}(\mathcal{O})$ eigenforms are zero at $x_{i}$. In our calculations, there are always exactly $r$ such $x_{i}$ 's, so these $x_{i}$ 's must be the fixed points of $\sigma_{N}$, and hence the sign (i.e., eigenvalue of $T_{N}$ ) for an eigenform $\varphi$ is +1 if $\varphi$ is nonzero at one of these $x_{i}$ 's and -1 otherwise.

When $d$ and $N$ are both large, the implementations of exact eigenvector calculations are slow in Magma and very slow in Sage. For example, consider $T_{2}$ with $N=1009$, which is represented by an $84 \times 84$ matrix. Its characteristic polynomial has irreducible factors of degrees 1, 37 and 46. Here exact calculations of eigenspaces took about 57 minutes of CPU time in Sage, and about 2 minutes of CPU time in Magma. Instead, we use numpy to compute approximate eigenvectors for prime $N<20000$, and thus compute numerical zeroes of eigenforms. (For $N=1009$, computing numerical eigenvectors with numpy only required about 0.09 seconds of CPU time.)

The issue, of course, with numerical eigenvector methods is that one needs to worry about how close the set of numerical zeroes matches the set of actual zeroes. While we can check that these counts do not always match exactly, they do appear to be very close. More precisely, for prime $N<20000$, we used a numerical version of the algorithm described above to numerically compute the fixed points of eigenforms, and thus numerically compute the root number of such eigenforms. Because this method always resulted in the correct number of fixed points, we believe that our numerical methods are properly detecting every trivial zero. In addition, comparing the numerical calculations with exact calculations for both small degree forms with prime $N<20000$ and all forms with prime $N<4000$ suggests that the number of numerical nontrivial zeroes we find is quite also close to the actual number of nontrivial zeroes. (Most numerical zeroes are accounted for by trivial zeroes or by nontrivial zeroes of small degree forms.) As further evidence that we are not picking up too many numerical nontrivial zeros which are not actual zeroes, consider the 1563 prime levels $N<20000$ such that $S(\mathcal{O})$ has exactly 2 Galois orbits. The eigenforms have no nontrivial zeroes in these levels by Theorem 4.4, and only 20 of these levels possess numerical nontrivial zeroes, and in each of these 20 levels there are exactly 2 spurious numerical nontrivial zeroes. (The smallest such level is 7351 , and the rest are over 10000.) 
We remark that in [Marb], we in fact computed a $T_{p}$ with no repeated eigenvalues for each prime $N<60000$. In principle we could use this to extend our calculations of numerical zeroes for $N>20000$, but the issue is that we do not have a good, practical way to either improve or estimate the accuracy of this numerical approach for a much larger range of $N$. The problem is that numerical methods are not good at distinguishing eigenspaces with close eigenvalues. In large levels it happens the numerical approximations for some nonzero entries are smaller (in absolute value) than the numerical approximations for entries which are actually zeroes, so one cannot simply adjust a numerical threshold to only detect true zeroes. Moreover, there is no simple way to increase precision in the numerical eigenvector methods in numpy, and in any case increasing the precision would significantly slow down the numerical methods.

For squarefree levels $N$, it is more complicated to determine which zeroes are trivial. Again, one could compute $T_{p}$ for each $p \mid N$ and use this to construct the signed graphs $\Sigma^{\varepsilon}$, which is feasible when all prime factors of $N$ are small. On the other hand, we have criteria to determine which sign patterns $\varepsilon$ have trivial zeroes, and can compute the dimension of each $M^{\varepsilon}(\mathcal{O})$ from the formulas for dimensions of Atkin-Lehner spaces in [Mar18]. Thus we can count how many forms have no trivial zeroes. We use this to determine exactly how many forms in $S(\mathcal{O})$ with no trivial zeroes are zerofree for squarefree levels $N<4000$.

5.2. Counting zeroes. For $N \in \mathrm{Sq}_{\text {odd }}$, fix a maximal quaternionic order $\mathcal{O}=\mathcal{O}_{N}$ of level $N$ in $B_{N}$. Let $S$ be a Hecke-invariant subspace of $M\left(\mathcal{O}_{N}\right)$. By the number of (trivial/nontrivial) zeroes for $S$ we mean the total number of (trivial/nontrivial) zeroes as we run over a basis of eigenforms for $S$.

Proposition 5.1. As $N \rightarrow \infty$ in $N \in \mathrm{Sq}_{\text {odd }}$, the number of trivial zeroes for $S\left(\mathcal{O}_{N}\right)$ is $O\left(N^{3 / 2+\epsilon}\right)$ for any $\epsilon>0$. This is essentially optimal in the sense that this number is not $O\left(N^{3 / 2-\epsilon}\right)$ for any $\epsilon>0$ when $N$ ranges over all of $\mathrm{Sq}_{\mathrm{odd}}$.

Proof. By Proposition 3.10, the maximum number of trivial zeroes of an eigenform $\varphi \in$ $S\left(\mathcal{O}_{N}\right)$ grows like $O\left(N^{1 / 2+\epsilon}\right)$. Since $\operatorname{dim} S\left(\mathcal{O}_{N}\right) \sim \frac{\varphi(N)}{12}=O(N)$, using this upper bound on every eigenform (up to scaling) in $S\left(\mathcal{O}_{N}\right)$ gives the first statement.

We know that the number of trivial zeroes cannot be $O\left(N^{3 / 2-\epsilon}\right)$ for any $\epsilon>0$ because Corollary 3.13 tells us it is not when we restrict to the subsequence of $N \in \mathrm{Sq}_{1}$.

For comparison, the total number of values (with multiplicity) of a basis of eigenforms for $S\left(\mathcal{O}_{N}\right)$ is $O\left(N^{2}\right)$. Precisely, there are $h-1$ linearly independent eigenforms in $S\left(\mathcal{O}_{N}\right)$, each of which have $h$ (not necessarily distinct) values $\varphi\left(x_{1}\right), \ldots, \varphi\left(x_{h}\right)$, giving $h^{2}-h$ total values where $h=1+\operatorname{dim} S_{2}^{\text {new }}(N) \sim \frac{\varphi(N)}{12}$.

We expect that most zeroes are trivial zeroes. Quantitatively, we predict the following.

Conjecture 5.2. As $N \rightarrow \infty$ along $\mathrm{Sq}_{r}$ for some $r$ or along $\mathrm{Sq}_{\text {odd }}$, the number of nontrivial zeroes for $S\left(\mathcal{O}_{N}\right)$ is $O\left(N^{1+\epsilon}\right)$ for any $\epsilon>0$.

This together with Theorem 1.4 suggests Conjecture 1.1(i).

Before we present some heuristics for this, we present some data. In Figure 1, we plot the total number of numerical nontrivial zeroes of $S\left(\mathcal{O}_{N}\right)$, for prime levels $N<20000$. In Figure 2 we also graph the total number of numerical nontrivial zeroes for all prime levels $\leq X$ when $X<20000$. These graphs indeed suggest that the number of nontrivial 
FiguRE 1. Number of nontrivial zeroes in each prime level $N$

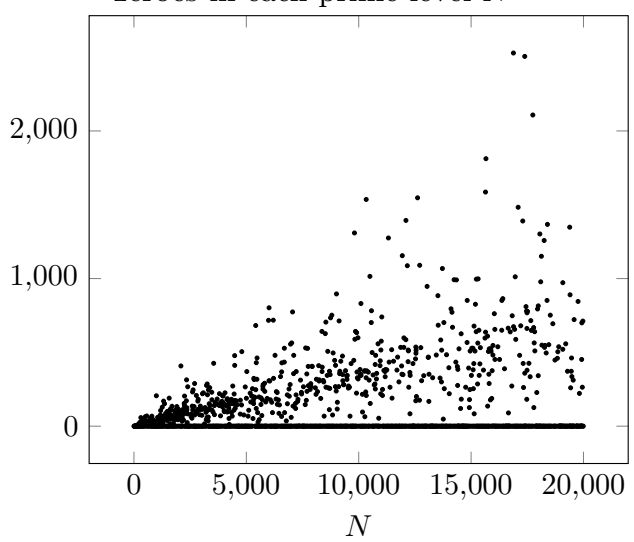

FiguRE 2. Number of nontrivial zeroes for prime levels $\leq X$

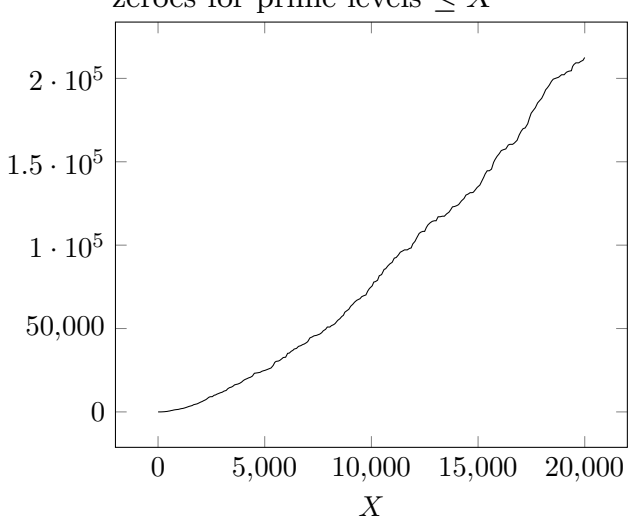

zeroes for $S\left(\mathcal{O}_{N}\right)$ grows not much faster than linearly in $N$, and that the same is true for the total number of nontrivial zeroes of all prime levels up to $X$. The latter quantity should not grow too much faster than the former quantity as we expect $100 \%$ of prime levels should have no nontrivial zeroes from Theorem 1.4. We note that out of the 2262 prime levels $N<20000$, there are numerical nontrivial zeroes in 682 of these levels.

In the rest of this section, we explain our main reasons for believing in Conjecture 5.2, which are interwined with Conjecture 1.2.

Say $\varphi \in S(\mathcal{O})$ has rationality field $K=K_{\varphi}$ of degree $d$. Normalize $\varphi$ so that $\varphi$ is integral and primitive, i.e., $\varphi\left(x_{1}\right), \ldots \varphi\left(x_{h}\right)$ all lie in $\mathfrak{o}_{K}$ and have no common factors besides units. Since $K$ is totally real, we have $d$ real embeddings, $\sigma_{1}, \ldots, \sigma_{d}: K \rightarrow \mathbb{R}$. This allows us to realize $\mathfrak{o}_{K}$ as a lattice $\Gamma \subset \mathbb{R}^{d}$ via $\alpha \mapsto\left(\sigma_{1}(\alpha), \ldots, \sigma_{d}(\alpha)\right)$. Similarly, let $\Lambda=\Gamma^{h} \subset \mathbb{R}^{d h}$ be the lattice obtained by a component-wise embedding. Then we may view $\varphi \in \Lambda$ as the lattice point corresponding to $\left(\varphi\left(x_{1}\right), \ldots \varphi\left(x_{h}\right)\right) \in \mathfrak{o}_{K}^{h}$.

For convenience, we will take the metric on $\mathbb{R}^{d h}$ to be the product of $\frac{1}{\sqrt{e_{i}}}$ times the standard metric on $\mathbb{R}^{d}$ over $1 \leq i \leq h$. Let $\lambda=\prod e_{i}^{-1 / 2}$. Then $\Lambda \subset \mathbb{R}^{d h}$ is a lattice with volume $\lambda \Delta_{K}^{h / 2}$ where $\Delta_{K}$ is the discriminant of $K$. Then the square length of $\varphi \in \Lambda$ is

$$
\langle\varphi, \varphi\rangle_{\Lambda}:=\sum_{\sigma_{j}} \sum_{i=1}^{h} \frac{1}{e_{i}} \sigma_{j}\left(\varphi\left(x_{i}\right)\right)^{2}=\operatorname{tr}_{K / \mathbb{Q}}(\varphi, \varphi) .
$$

We would like to model $\varphi$ as a random lattice point in $\Lambda$ on the sphere of radius $\langle\varphi, \varphi\rangle_{\Lambda}^{1 / 2}$.

For a hypersphere $S^{n-1}$ in $\mathbb{R}^{n}$, and coordinate functions $X_{1}, \ldots, X_{d}$ of random point on $S^{n-1}$, a result of Poincaré tells us that the distribution of $\left(X_{1}, \ldots, X_{d}\right)$ tends to a $d$ dimensional normal distribution as $n \rightarrow \infty$-in fact $d$ is also allowed to vary, provided that $d$ grows slower than $n$, i.e., $d=o(n)$ [DF87]. Consequently, viewing $\varphi \in \Lambda$ as a randomly chosen lattice point on some hypersphere of radius $R$ in $\mathbb{R}^{d h}$, a given value $\varphi\left(x_{i}\right) \in \mathfrak{o}_{K} \subset$ $\mathbb{R}^{d}$ should be distributed approximately like a $d$-dimensional normal distribution when $N$ is large. Note that $d \leq h$ so $d=o(n)$ where $n=d h$. (See Figures 5 and 6 below for two numerical examples when $d=1,2$.) As long as the radius $R$ is not too small with respect to $\Delta_{K}$, the probability that $\varphi\left(x_{i}\right)=0$ will decrease as $d$ increases, essentially 
TABLE 1. Degree histograms about nontrivial zeroes for prime levels $N<4000$

\begin{tabular}{r|rrrrrrrrrr}
$d$ & 1 & 2 & 3 & 4 & 5 & 6 & 7 & 8 & 9 & $\geq 10$ \\
\hline tot \#orbits & 179 & 133 & 57 & 25 & 19 & 10 & 18 & 3 & 12 & 983 \\
\#orbits w/nontriv 0's & 152 & 110 & 35 & 14 & 5 & 2 & 2 & 0 & 2 & 0 \\
\#nontriv 0's & 9730 & 5896 & 1860 & 864 & 210 & 120 & 28 & 0 & 27 & 0 \\
\#nontriv 0's/all 0's & 0.873 & 0.713 & 0.543 & 0.451 & 0.214 & 0.175 & 0.035 & 0 & 0.032 & 0 \\
\#nontriv 0's/all vals & 0.396 & 0.177 & 0.123 & 0.101 & 0.046 & 0.042 & 0.007 & 0 & 0.006 & 0
\end{tabular}

because the number of lattice points $\mathfrak{o}_{K}$ in the ball of radius $R$ is increasing. While we do not have precise heuristics about $\langle\varphi, \varphi\rangle_{\Lambda}=R^{2}$, but numerically it appears to increase suitably rapidly with $d$ and $\Delta_{K}$.

This idea, together with data and Corollary 4.3, is what leads us to believe in Conjecture 1.2, i.e., that forms with smaller degree are more likely to have zeroes. In Table 1 we tabulate the nontrivial exact zeroes by degree among prime levels $N<4000$, which supports this conjecture. The first row of data tells us how many Galois orbits of eigenforms of degree $d$ there are in this range, and the second row counts how many of these orbits have nontrivial zeroes. The third row counts the total number of nontrivial zeroes coming from degree $d$ forms in this range, the fourth row gives the proportion of all zeroes which are nontrivial zeroes, and the final row gives the proportion all values (with multiplicity) which are nontrivial zeroes. (In the third row, we count each eigenform in a Galois orbit separately; in the last two rows the proportion is the same looking at individual eigenforms or looking at Galois orbits.) While this range $N<4000$ is rather limited, the key point of this table is that it indicates that the frequency of nontrivial zeroes tends to decrease in $d$.

Moreover, from [Marb], we have the expectation that $100 \%$ of the time that an eigenspace $S^{\varepsilon}\left(\mathcal{O}_{N}\right)$ has multiple Galois orbits it is because of the existence of degree 1 forms. In summary, most of the small Galois orbits consist of degree 1 forms, and degree 1 forms are more likely than higher degree forms to have zeroes. This leads to the following more precise conjecture.

Conjecture 5.3. As $N \rightarrow \infty$ along $\mathrm{Sq}_{r}$ for some odd $r$, or along $\mathrm{Sq}_{\text {odd }}, 100 \%$ of nontrivial zeroes of $S\left(\mathcal{O}_{N}\right)$ come from degree 1 forms.

Remark 5.4. We similarly expect that if one restricts to forms of degree $\geq 2$, that $100 \%$ of non-trivial zeroes come from degree 2 forms. More generally, we expect a similar sort of phenomenon that degree $d$ forms tend to contribute most of the zeroes among degree $\geq d$ forms. However, we are hesitant to formulate a precise conjecture about this in general, as it is not even clear if for fixed $d$ there should be infinitely many forms of degree $d$ with prime level. See [Marb] for a brief discussion of this latter issue.

In Figure 3, we present some data on the proportion of numerical nontrivial zeroes of prime levels $\leq X$ which are accounted for by the (actual) nontrivial zeroes of degree 1 forms. Note that this proportion appears to be slowly increasing. While it is not clear from the graph alone whether this proportion is tending to 1, we expect that the slow rate of increase is due to the existence of many small degree forms in small levels (cf. [Marb]). In addition, we believe the numerical nontrivial zeroes may be a slight overcount of the actual nontrivial zeroes for $N$ large. 
FiguRE 3. Proportion of nontrivial zeroes for prime levels $\leq X$ coming from degree 1 forms

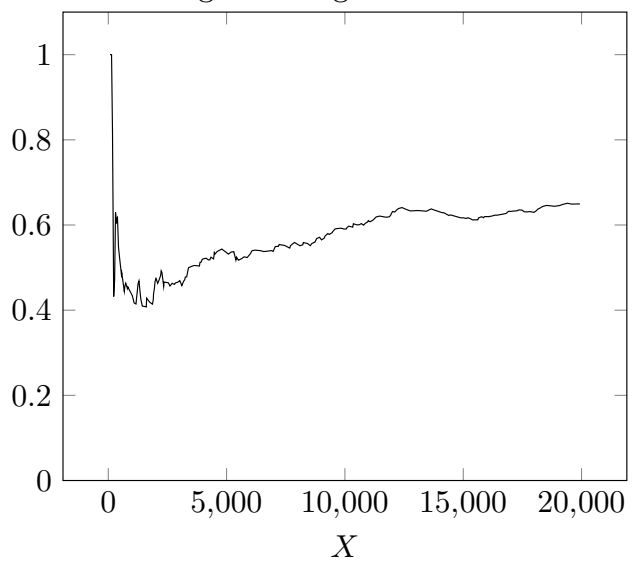

Figure 4. Proportion of values of degree 1 forms (excluding trivial zeroes) which are 0

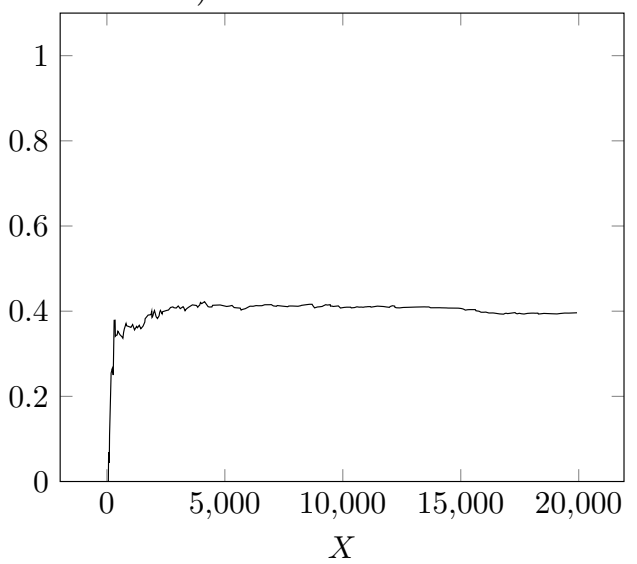

At the least, we expect that almost all nontrivial zeroes are accounted for by small degree forms. Further, we expect the number of forms of small degree in a given squarefree level $N$ to be $O\left(N^{\varepsilon}\right)$ for any $\varepsilon>0$. (In fact, it would not be that surprising if the number of forms of small degree of a given prime level $N$ is $O(1)$ - e.g., see the data in [Marb].) These two expectations suggest Conjecture 5.2.

Remark 5.5. One might further ask if the number of nontrivial zeroes for $S(\mathcal{O})$ is actually $o(N)$. This is certainly not clear from the limited amount of data we have, but we suspect this is possible for the following reasons. First, we expect $100 \%$ of nontrivial zeroes to come from small degree forms. Second, if $\varphi$ is degree $d$, we expect the proportion of $\varphi\left(x_{i}\right)$ 's which vanish nontrivially can be modeled with a close-to-normal $d$-dimensional discrete distribution with variance depending on some radius $R=\langle\varphi, \varphi\rangle_{\Lambda}^{1 / 2}$. It appears that, for fixed $d,\langle\varphi, \varphi\rangle_{\Lambda}^{1 / 2}$ tends to increase with $N$. If the rate of increase is strictly faster than linear, then as $N$ increases the proportion of $\varphi\left(x_{i}\right)$ 's which are nontrivially 0 should tend to 0 , which would suggest the total number of nontrivial zeroes for $S\left(\mathcal{O}_{N}\right)$ (and thus also for a single form in $S\left(\mathcal{O}_{N}\right)$ ) is $o(N)$. In Figure 4, we graph the proportion of nontrivial zeroes among values of degree 1 forms (excluding trivial zeroes) of prime levels $\leq X$. We suspect that this proportion may be decreasing very slowly, similar to how the average rank of elliptic curves appears to converge very slowly. Indeed, using the proportion of zeroes of a form as a proxy for the probability of vanishing $L$-values as mentioned in the introduction, the expectation that $0 \%$ of elliptic curves of should have rank $\geq 2$ also suggests that the proportion of values which are nontrivial zeroes over all degree 1 forms should be 0 .

5.3. Values of quaternionic modular forms. One phenomenon we observed is that the values of degree 1 (primitive integral) eigenforms tend to be quite small. Of the 529 normalized degree 1 eigenforms of prime level $N<20000$, only 55 take on a value of size $\geq 5$. Of these, only 6 have absolute values $\geq 10$ - there are 2 forms with maximum absolute value 11 (in levels 9473 and 16193) and 4 with maximum absolute value 12 (2 in level 8747 and 1 each in levels 13723 and 17333). Of these 6 forms, only the one 
FiguRE 5. Histogram of values of the degree 1 form of prime level 17333

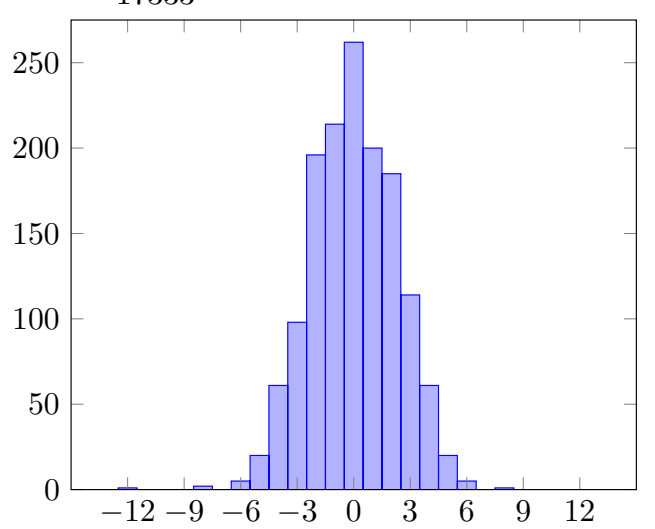

Figure 6. Histogram of values for a degree 2 form of prime level 16889

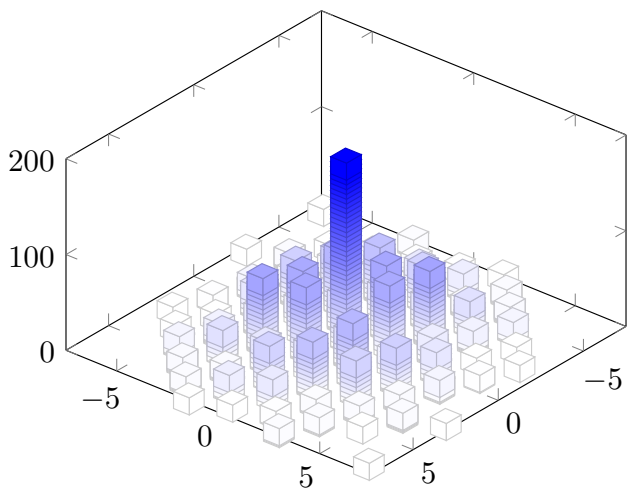

in level 13723 has root number -1. In Figure 5, we present a histogram of the values of the unique-up-to-scalars degree 1 eigenform of level 17333. The general shape of the histogram seems to be typical for degree 1 eigenforms - the values tend to be small and cluster roughly symmetrically around 0 following a roughly normal-shaped distribution. (The histogram will be completely symmetric in the case of root number -1 .)

Consider a degree $d$ eigenform $\varphi$, normalized so its values are in $K=K_{\varphi}$, as a lattice point in $K^{h} \hookrightarrow\left(\mathbb{R}^{d}\right)^{h}$ as explained above. We expect that the $(d+1)$-dimensional histograms of values of degree $d$ eigenforms will have an analogous shape of clustering about 0 and then rapidly tapering off, similar to a $d$-dimensional normal distribution. In Figure 6, we plot a histogram of the values the unique (up to scaling and Galois conjugation) degree 2 form of prime level 16889, which has has root number $-1, h=1408$ total values, and 182 zeroes (72 trivial, 110 nontrivial). The most common nonzero value occurs 65 times. So even if we exclude trivial zeroes, the distribution of values looks similar to a 2-dimensional normal distribution.

5.4. Zerofree forms. In Figures 7 and 8, we plot the proportion of zero-free forms among eigenforms with no trivial zeroes in levels $N \leq X$ for $X<4000$. The former plot restricts to $N$ prime and the latter plot restricts to $N$ non-prime. These data indeed suggest that this proportion tends to $100 \%$, in line with what we expect from Conjecture 1.1(ii). Note that convergence appears to be slower in the non-prime level case, which is to be expected in light of Conjecture 1.2 as there tend to be more small Galois orbits when there are more Atkin-Lehner eigenspaces (cf. [Marb]).

\section{Periods And $L$-VAlues}

Consider a quadratic field $K=\mathbb{Q}(\sqrt{-D})$ of discriminant $-D$. Identify the ideal class group $\mathrm{Cl}(K)$ with $K^{\times} \backslash \hat{K}^{\times} / \hat{\mathfrak{o}}_{K}^{\times}$. It is well known that $K$ embeds in $B$ if and only if $K$ is imaginary quadratic and $K_{p}$ is non-split (i.e., $\left(\frac{-D}{p}\right) \neq 1$ ) for all $p \mid N$. Assume $K$ embeds into $B$.

For an ideal $\mathcal{J}$ in $K$ (resp. $B$ ) or an idele $\alpha$ in $\hat{K}^{\times}$(resp. $\hat{B}^{\times}$), we sometimes denote the corresponding $\mathfrak{o}_{K^{-}}$(resp. $\mathcal{O}$-)ideal class by $[\mathcal{J}]$ or $[\alpha]$. 
Figure 7. Proportion of zero-free forms for prime level

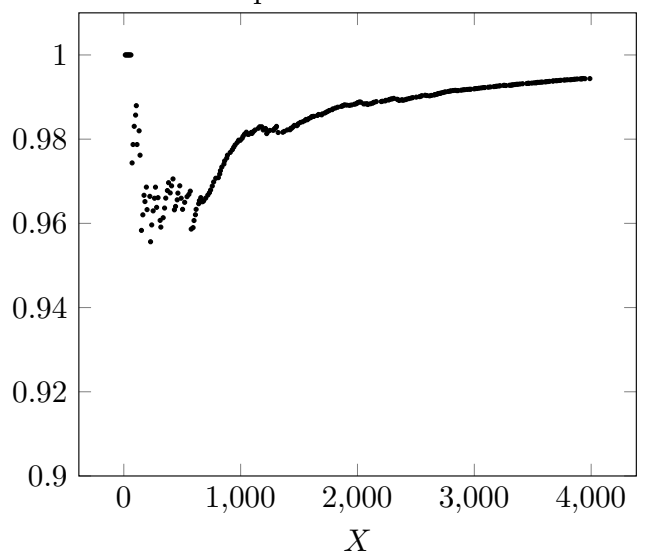

FiguRe 8. Proportion of zero-free forms for non-prime level

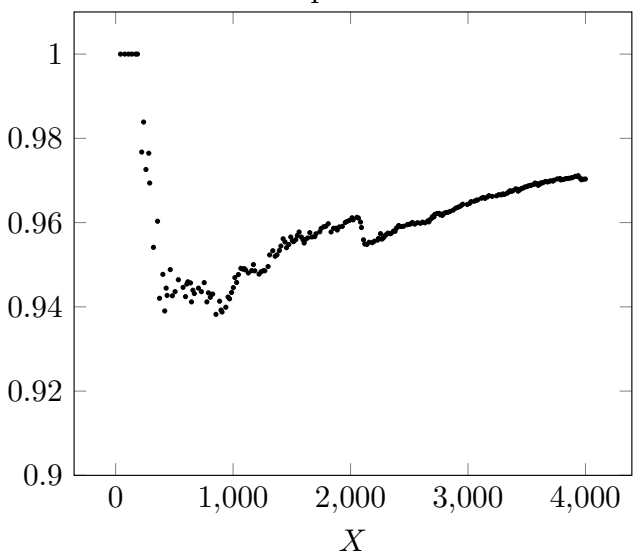

6.1. The ideal class map. Let $\mathcal{O}$ be a maximal order in $B$ such that $\mathfrak{o}_{K}$ embeds into $\mathcal{O}$. There is always some such $\mathcal{O}$. Denote by $\iota: K \rightarrow B$ an embedding such that $\iota\left(\mathfrak{o}_{K}\right) \subset \mathcal{O} .^{3}$ Then this embedding induces a map (necessarily not injective if $h_{K}>h$ ) of ideal classes $\iota_{*}: \mathrm{Cl}(K) \rightarrow \mathrm{Cl}(\mathcal{O})$ given by $\iota_{*}([t])=[\iota(t)]$ for $t \in \hat{K}^{\times}$. This is well defined as $\iota\left(K^{\times} t \hat{\mathfrak{o}}_{K}^{\times}\right) \subset B^{\times} \iota(t) \hat{\mathcal{O}}^{\times}$. In what follows, we fix our embedding $\iota: K \rightarrow B$, and will simply write $t$ for $\iota(t)$, where $t \in \hat{K}^{\times}$.

The precise behavior of these ideal class maps is mysterious and connected to deep problems in arithmetic, such as values of $L$-functions. However, we can identify a few elementary properties of these maps. First, note that $\iota_{*}$ is not just a map of sets, but a map of pointed sets, where the distinguished points for $\mathrm{Cl}(K)$ and $\mathrm{Cl}(\mathcal{O})$ are the ideal classes of $\mathfrak{o}_{K}$ and $\mathcal{O}$, respectively. In particular, if $K$ has class number 1 , then $\iota_{*}$ does not depend upon the choice of $\iota$ (and in this case, there is only one choice for $\mathcal{O}$ up to isomorphism).

Remark 6.1. If $K=\mathbb{Q}(i)$ or $\mathbb{Q}(\sqrt{-3})$, then $\mathfrak{o}_{K}^{\times}$has order 4 or 6 . If $N>2$ is prime and $K$ embeds in $B$, then there is a unique $i$ such that $\left[\mathfrak{o}_{K}^{\times}: \mathbb{Z}^{\times}\right.$] divides $e_{i}$ (e.g., see [Gro87, Table 1.3]). Consequently, there is a unique maximal order $\mathcal{O} \subset B$ up to isomorphism such that $\mathcal{O}^{\times}$contains a 4 th or 6 th root of unity. This provides one way to specify exactly which $\mathcal{O}$ possess an embedding of $\mathfrak{o}_{K}$ in these special cases.

Next we give two slightly more interesting properties. For $d \mid N$, write $\sigma_{d}(x)=\prod_{p \mid d} \sigma_{p}(x)$.

Proposition 6.2. The class map satisfies $\iota_{*}\left(t^{-1}\right)=\sigma_{N}\left(\iota_{*}(t)\right)$ for $t \in \mathrm{Cl}(K)$.

Proof. Consider the orthogonal decomposition (with respect to the norm form) $B=$ $K \oplus j K$, where $j \in B^{\times}$. Let $\varpi_{N} \in B^{\times}$be an element of reduced norm $N$. We claim there is a rational element $\alpha \in j K \cap \varpi_{N} \hat{\mathcal{O}}^{\times}$. Assuming there is such an $\alpha$, note conjugation by $\alpha$ acts on $K$ as the standard (Galois) involution $a \mapsto \bar{a}$ since $\alpha \in j K$. Then for $t \in \hat{K}^{\times}$,

\footnotetext{
${ }^{3}$ Eichler showed that, summing over maximal ideals $\mathcal{O}$, the total number of such embeddings up to conjugation by units is essentially $h_{K}$. So in general, there are many choices for such an embedding. Moreover $\mathfrak{o}_{K}$ embeds in every maximal $\mathcal{O}$ when $h_{K}$ is sufficiently large relative to $N$.
} 
we have

$$
B^{\times} \bar{t} \hat{\mathcal{O}}^{\times}=B^{\times} \alpha^{-1} t \alpha \hat{\mathcal{O}}^{\times}=B^{\times} t \varpi_{N} \hat{\mathcal{O}}^{\times}=\sigma_{N}\left(B^{\times} t \hat{\mathcal{O}}^{\times}\right) .
$$

Since $[\bar{t}]=\left[t^{-1}\right]$ in $\mathrm{Cl}(K)$, we get that $\iota_{*}\left(\left[t^{-1}\right]\right)=\sigma_{N}\left(\iota_{*}([t])\right)$.

So it suffices to prove the existence of an $\alpha$ as above. For this, we claim that there exists a prime $q \nmid D N$ split in $K$ such that $B \simeq\left(\frac{-D,-N q}{\mathbb{Q}}\right)$. That is, we want to show the existence of a prime $q \nmid D N$ split in $K$ such that $\left(\frac{-D,-N q}{\mathbb{Q}}\right)$ has discriminant $N$, i.e., such that $\left(\frac{-D,-N q}{\mathbb{Q}_{p}}\right)$ is a division algebra if and only if $p \mid N$. In fact, since $\left(\frac{-D,-N q}{\mathbb{Q}}\right)$ is necessarily definite, it must be ramified at an odd number of finite primes, so we may restrict this latter condition to odd primes $p$.

Standard splitting criteria for quaternion algebras imply the following result.

Lemma 6.3. Suppose $p$ is odd. Assume $a, b \in \mathbb{Z}$ are nonzero and squarefree with $v_{p}(a) \leq$ $v_{p}(b)$. Then $\left(\frac{a, b}{\mathbb{Q}_{p}}\right)$ is a division algebra if and only if (1) $p \nmid a, p \mid b$ and $\left(\frac{a}{p}\right)=-1$; or (2) $p \mid a$ and $-b / a$ is a nonsquare $\bmod p$.

Assume $q$ is a prime not dividing $D N$. First, we want $q$ to satisfy (i) $\left(\frac{-D}{q}\right)=+1$ for $q$ to be split in $K$, and by the above lemma, this also means that $\left(\frac{-D,-N q}{\mathbb{Q}}\right)$ is split at $q$-i.e., the localization at $q$ is isomorphic to $M_{2}\left(\mathbb{Q}_{q}\right)$-as desired. Also, if $p \nmid D N q$ is odd, then $\left(\frac{-D,-N q}{\mathbb{Q}}\right)$ is split at $p$ by the lemma.

Next consider a prime $p$ such that $p \mid N$ but $p \nmid D$. Then $\left(\frac{-D}{p}\right)=-1$ since $K$ embeds in $B$, which means that $\left(\frac{-D,-N q}{\mathbb{Q}}\right)$ is divison at $p$, also as desired.

Now consider an odd prime $p$ such that $p \mid D$ but $p \nmid N$. Then $\left(\frac{-D,-N q}{\mathbb{Q}}\right)$ is split at $p$ if and only if (ii) $\left(\frac{q}{p}\right)=\left(\frac{-N}{p}\right)$.

Finally, consider an odd prime $p \mid \operatorname{gcd}(D, N)$. Then $\left(\frac{-D,-N q}{\mathbb{Q}}\right)$ is division at $p$ if and only if (iii) $\left(\frac{c q}{p}\right)=-1$ where $c$ is an integer equivalent to $N / D \bmod p$.

By Dirichlet's theorem on primes in progressions, there exists a prime $q$ satisfying (i), (ii) and (iii) as above, which then gives us a $q$ as claimed. Consequently, we may take our orthogonal decomposition $B=K \oplus j K$ above with $j^{2}=-N q$, and then we may take $\alpha=j a$ where $a \in K$ is an element of norm $q^{-1}$.

Corollary 6.4. Suppose $t \in \mathrm{Cl}(K)$ has order $\leq 2$. Then $\iota_{*}(t)$ is a fixed point of $\sigma_{N}$.

Note that this gives an alternative proof of Lemma 3.4. Here is another result in the spirit of this corollary.

Proposition 6.5. Let $d=D$ if $4 \nmid D$ and $d=\frac{D}{4}$ if $4 \mid D$. Suppose $d \mid N$. Then for $t \in \mathrm{Cl}(K), \sigma_{d}\left(\iota_{*}(t)\right)=\iota_{*}(t)$, i.e., the image of $\iota_{*}$ lies in the set of fixed points of $\sigma_{d}$.

Proof. Let $\alpha=\iota(\sqrt{-d})$. Then $\alpha_{p} \in \mathcal{O}_{p}^{\times}$for $p \nmid d$ and $\alpha_{p}$ is a uniformizer $\varpi_{B_{p}}$ in $B_{p}$ for $p \mid d$. Thus for $t \in \hat{K}^{\times}, t$ commutes with $\alpha \in K$, so

$$
B^{\times} t \hat{\mathcal{O}}^{\times}=B^{\times} \alpha^{-1} t \alpha \hat{\mathcal{O}}^{\times}=B^{\times} t \prod_{p \mid d} \varpi_{B_{p}} \hat{\mathcal{O}}^{\times},
$$

which proves the assertion.

We remark that, when $d=p$, the conclusion that $\sigma_{p}$ has fixed points in this situation provides a different proof of part of Lemma 3.9. 
Here is one more elementary result in the case that $K$ and $B$ have joint ramification.

Proposition 6.6. Suppose $p \mid \operatorname{gcd}(D, N)$. Let $\mathcal{J}_{p}$ denote the class of an ideal in $\mathfrak{o}_{K}$ of norm $p$. Multiplication by $\mathcal{J}_{p}$ acts on $\mathrm{Cl}(K)$ as an involution, which we also denote by $\sigma_{p}$. Then $\iota_{*}: \mathrm{Cl}(K) \rightarrow \mathrm{Cl}(\mathcal{O})$ respects the action of $\sigma_{p}$, i.e., $\iota_{*}\left(\sigma_{p}(t)\right)=\iota_{*}\left(t \mathcal{J}_{p}\right)=\sigma_{p}\left(\iota_{*}(t)\right)$.

Proof. This is clear, as a uniformizer for $K_{p}$ is also a uniformizer for $B_{p}$.

Other properties of the ideal class map require much deeper understanding of arithmetic. Notably, Michel uses subconvexity of $L$-functions to show that the ideal class maps are equidistributed in a suitable sense (for fixed $B$ and varying $K$, as $D \rightarrow \infty$ ) [Mic04, Theorem 10]. One application of equidistribution of the ideal class map is to the existence of nonvanishing twisted central $L$-values [MV07] - see also Remark 6.13.

Remark 6.7. Here is an unexpected (at least to us) consequence of equidistribution combined with Corollary 6.4. The latter says that if $K \subset B$ has one class per genus, i.e., $\mathrm{Cl}(K)$ has exponent $\leq 2$, then the image of the class map $\iota_{*}$ is contained in the fixed points of $\sigma_{N}$. However, assuming $\operatorname{dim} S_{2}^{-N}(N) \neq 0$ (e.g., if $N>71$ is prime [Mar18, Proposition 4.2]), then not all elements of $\mathrm{Cl}(\mathcal{O})$ are fixed by $\sigma_{N}$. Thus the ideal class map is not surjective. But equidistribution of this map means that for $D$ sufficiently large, $\mathrm{Cl}(K) \rightarrow \mathrm{Cl}(\mathcal{O})$ is surjective. Consequently, there can only be finitely many one-class-pergenus fields $K$ embedding in $B$. Even though the much stronger result that there are at most 66 imaginary quadratic one-class-per-genus fields is known [Wei73], this argument is interesting, as to our knowledge it is rather different than existing proofs of finiteness results for certain class group structures. Of course, deep facts about $L$-functions (subconvexity) still underlie this argument.

6.2. Toric periods. Let $\chi$ be a character of $\mathrm{Cl}(K)$. We fix Haar measures on $\hat{K}^{\times}$and $\hat{\mathbb{Q}}^{\times}$such that the quotient $K^{\times} \hat{\mathbb{Q}}^{\times} \backslash \hat{K}^{\times} / \hat{\mathfrak{o}}_{K}^{\times} \simeq \mathrm{Cl}(K)$ has measure $h_{K}$.

Define the period integral $P_{K, \chi}: M(\mathcal{O}) \rightarrow \mathbb{C}$ by

$$
P_{K, \chi}(\varphi)=\int_{K^{\times} \hat{\mathbb{Q}}^{\times} \backslash \hat{K}^{\times}} \varphi(t) \chi^{-1}(t) d t=\sum_{t \in \mathrm{Cl}(K)} \varphi\left(\iota_{*}(t)\right) \chi^{-1}(t) .
$$

In the case that $\chi$ is trivial we simply denote $P_{K, \chi}$ by $P_{K}$.

For $\pi$ an automorphic representation occurring in $S(\mathcal{O})$ with corresponding cuspidal automorphic representation $\pi^{\prime}$ of $\mathrm{GL}_{2}\left(\mathbb{A}_{\mathbb{Q}}\right)$, and $\varphi \in \pi^{\hat{\mathcal{O}}^{\times}}$, Waldspurger [Wal85] proved a formula of the form

$$
\left|P_{K, \chi}(\varphi)\right|^{2}=c(\varphi, K, \chi) L\left(\frac{1}{2}, \pi_{K}^{\prime} \otimes \chi\right) .
$$

Here $\pi_{K}^{\prime}$ denotes the base change of $\pi^{\prime}$ to $\mathrm{GL}_{2}\left(\mathbb{A}_{K}\right)$. If $\chi$ is trivial, then $L\left(s, \pi_{K}^{\prime}\right)=$ $L\left(s, \pi^{\prime}\right) L\left(s, \pi^{\prime} \otimes \eta_{D}\right)$, where $\eta_{D}$ is the quadratic Dirichlet associated to $K / \mathbb{Q}$.

For simplicity in what follows, for an eigenform $\varphi \in \pi \cap S(\mathcal{O})$ as above, we write $L(s, \varphi)=L\left(s, \pi^{\prime}\right), L\left(s, \varphi_{K} \otimes \chi\right)=L\left(s, \pi_{K}^{\prime} \otimes \chi\right)$, etc., and similarly for $\varepsilon$-factors.

In particular, $P_{K, \chi}(\varphi) \neq 0$ implies the twisted central $L$-value $L\left(\frac{1}{2}, \varphi_{K} \otimes \chi\right) \neq 0$. The converse is not true as $c(\varphi, K, \chi)$ may be 0 . Indeed, by Tunnell [Tun83], $P_{K, \chi}$ is identically 0 on $\pi$ (the period is well defined for any smooth $\varphi \in \pi$, not just $\varphi \in S(\mathcal{O})$ ) unless the local $\varepsilon$-factors satisfy $\varepsilon_{v}\left(\frac{1}{2}, \varphi_{K} \otimes \chi\right)=+1$ if and only if $B_{v}$ is ramified. Note that this local $\varepsilon$-factor condition forces the global root number of $\varphi_{K} \otimes \chi$ to be +1 . 
Precisely, the following holds. For an eigenform $\varphi \in M(\mathcal{O})$ and $d \mid N$, let $\varepsilon_{d}(\varphi)$ be the $T_{d}$-eigenvalue of $\varphi$. For $p \mid D$, let $\varpi_{K_{p}}$ denote a uniformizer for $K_{p}$.

Proposition 6.8. Let $\varphi \in S(\mathcal{O})$ be an eigenform and $\chi$ be a character of $\mathrm{Cl}(K)$. Then $P_{K, \chi}(\varphi)=0$ unless:

(i) $\varepsilon_{N}(\varphi)=+1$ if $\chi$ is quadratic; and

(ii) $\varepsilon_{p}(\varphi)=\chi_{p}\left(\varpi_{K_{p}}\right)$ for all $p \mid \operatorname{gcd}(N, D)$.

Moreover, assuming (ii), $L\left(\frac{1}{2}, \varphi_{K} \otimes \chi\right) \neq 0 \Longleftrightarrow P_{K, \chi}(\varphi) \neq 0$.

Condition (ii) corresponds to Tunnell's $\varepsilon$-factor criterion. See Remark 6.9 about (i).

Proof. First suppose $\chi$ is quadratic. Then by Proposition 6.2,

$$
\varepsilon_{N}(\varphi) P_{K, \chi}(\varphi)=P_{K, \chi}\left(T_{N}(\varphi)\right)=\sum \varphi\left(\sigma_{N}\left(\iota_{*}(t)\right)\right) \chi^{-1}(t)=\sum \varphi\left(\iota_{*}(t)\right) \chi(t)=P_{K, \chi}(\varphi) .
$$

In particular, $P_{K, \chi}(\varphi)=0$ unless (i) holds.

Similarly, by Proposition 6.6, one sees that $\varepsilon_{p}(\varphi) P_{K, \chi}(\varphi)=\chi_{p}\left(\varpi_{K_{p}}\right) P_{K, \chi}(\varphi)$ for $p \mid \operatorname{gcd}(N, D)$. Hence $P_{K, \chi}(\varphi)=0$ unless (ii) holds.

Now we explain the final part. The above integral definition of $P_{K, \chi}(\psi)$ makes sense for smooth elements in $\psi \in \pi$, so we may view $P_{K, \chi}$ as an element of $\operatorname{Hom}_{\mathbb{A}} \times(\pi, \chi)$. Via the factorization of $\pi=\otimes \pi_{v}$, this global Hom space can only be nonzero if the local Hom spaces $\operatorname{Hom}_{K_{v}^{\times}}\left(\pi_{v}, \chi_{v}\right)$ are all nonzero. It follows from Jacquet's relative trace formula approach to Waldspurger's theorem that $L\left(\frac{1}{2}, \pi_{K} \otimes \chi\right) \neq 0$ if and only if $P_{K, \chi}$ is not identically zero on $\pi$ (the main result of [Jac87], with a gap fixed in [JC01] - see p. 41 of the latter paper). From the work of Gross and Prasad on test vectors, $P_{K, \chi}$ is not identically 0 on $\pi$ if and only if $P_{K, \chi}(\varphi) \neq 0$ if $\varphi$ is a nonzero element of $\pi^{\hat{\mathcal{O}}^{\times}}$ [GP91, Proposition 2.6].

It remains to check that (ii) guarantees each local Hom space is nonzero. While one could use the $\varepsilon$-factor criterion in [Tun83] to check this, it is simple to give a direct argument. The local archimedean Hom space is nonzero since $\pi_{\infty}$ and $\chi_{\infty}$ are trivial. For finite $p \nmid N, \pi_{p}$ is an unramified principal series and thus the local Hom space $\operatorname{Hom}_{K_{p}^{\times}}\left(\pi_{p}, \mu_{p}\right) \neq 0$ for any character $\mu_{p}$ of $K_{p}$ with trivial central character.

Now suppose $p \mid N$. Then $\pi_{p}$ is 1-dimensional, either trivial if $\varepsilon_{p}(\varphi)=1$ or $\eta_{p} \circ$ det if $\varepsilon_{p}(\varphi)=-1$, where $\eta_{p}$ is the unramified quadratic character of $\mathbb{Q}_{p}^{\times}$. In either case, the local Hom space $\operatorname{Hom}_{K_{p}^{\times}}\left(\pi_{p}, \chi_{p}\right)$ being nonzero simply means that $\left.\pi_{p}\right|_{K_{p}^{\times}}=\chi_{p}$. Note $\left.\pi_{p}\right|_{K_{p}^{\times}}$ and $\chi_{p}$ are both characters of $K_{p}^{\times} / \mathfrak{o}_{K_{p}}^{\times} \mathbb{Q}_{p}^{\times}$. If $K_{p} / \mathbb{Q}_{p}$ is unramified, then this quotient is trivial and $\left.\pi_{p}\right|_{K_{p}^{\times}}=\chi_{p}$ holds automatically. If $K_{p} / \mathbb{Q}_{p}$ is ramified, then the quotient has order 2 and is generated by a uniformizer $\varpi_{K_{p}}$, in which case (ii) is equivalent to both characters agreeing on $K_{p}^{\times}$.

Remark 6.9. While this type of result is clear to experts and has often been used, to our knowledge this is the first direct proof for why the periods must vanish when (i) or (ii) fails to hold. Rather the standard argument for vanishing of periods in such situations is either because the local Hom space is zero or that a root number condition forces an $L$-value to vanish. For instance, regarding (i), when $\chi$ is quadratic, the automorphic induction to $\mathrm{GL}_{2}\left(\mathbb{A}_{\mathbb{Q}}\right)$ is a nontrivial isobaric sum and so $L\left(s, \varphi_{K} \otimes \chi\right)$ factors into 2 degree $2 L$-functions over $\mathbb{Q}$. When $\chi=1$, we see $L\left(s, \varphi_{K}\right)=L(s, \varphi) L\left(s, \varphi \otimes \eta_{K / \mathbb{Q}}\right)$ 
where $\eta_{K / \mathbb{Q}}$ is the quadratic character over $\mathbb{Q}$ associated to $K / \mathbb{Q}$. From this it is not hard to see that the two $L$-functions on the right have root number +1 if and only if $\varphi$ does assuming (ii). When $\chi$ is nontrivial quadratic, it is less obvious that the root numbers of the factored $L$-functions match the root number of $L(s, \varphi)$. We expect one can check that this is so, but have not attempted to do so and have not seen this in the literature. Accordingly, while the $\chi=1$ case of (i) is well known, we are unsure whether (ii) was previously known for $\chi$ of order 2 .

In the following results, recall that we assume $\mathfrak{o}_{K} \subset \mathcal{O}$.

Theorem 6.10. Suppose $K$ has one class per genus. Let $\chi$ be a character of $\mathrm{Cl}(K), \varepsilon$ be a sign pattern for $N$, and $\varphi \in S^{\varepsilon}(\mathcal{O})$ be an eigenform. Assume one of the following:

(1) $h_{K}=1$; or

(2) for each $p \mid D$, we have that $p \mid N$ and $\chi_{p}\left(\varpi_{K_{p}}\right)=\varepsilon_{p}$.

Then $L\left(\frac{1}{2}, \varphi_{K} \otimes \chi\right) \neq 0 \Longleftrightarrow \varphi(1) \neq 0$.

Proof. By the previous result, it suffices to show that $P_{K, \chi}(\varphi)$ is a non-zero multiple of $\varphi(1)$.

Case (1) is clear, as then $x_{0}:=\iota_{*}\left(\left[\mathfrak{o}_{K}\right]\right)$ is a fixed point of $\sigma_{N}$ and thus $P_{K}(\varphi)=$ $\varphi\left(x_{0}\right)=\varphi(1)$. (Necessarily $\chi=1$ here.)

Now assume (2). For $p \mid D$, let $\hat{\varpi}_{K_{p}} \in \hat{K}$ denote an element whose local components $\left(\hat{\varpi}_{K_{p}}\right)_{v} \in \mathfrak{o}_{K, v}^{\times}$are integral units for all finite primes $v$ of $K$ not lying above $p$, and whose local component is a uniformizer at the prime above $p$. Then $\mathrm{Cl}(K)$ is generated by the $\hat{\varpi}_{K_{p}}$ 's, i.e., the ideals of absolute norm $p$, for $p \mid D$. Thus for any $t \in \mathrm{Cl}(K)$, we may choose a representative in $\hat{K}^{\times}$of the form $\prod \hat{\varpi}_{K_{p}}$ for some subset of $\{p: p \mid D\}$. By Proposition 6.6, we see that $\varphi(t)=\varphi\left(\prod \hat{\varpi}_{K_{p}}\right)=\prod \varepsilon_{p}(\varphi) \cdot \varphi(1)=\chi(t) \varphi(1)$. Since $\mathrm{Cl}(K)$ has exponent $2, \chi$ must be quadratic. Thus we deduce $P_{K, \chi}(\varphi)=h_{K} \varphi(1)$.

Corollary 6.11. Fix $\mathcal{O} \subset B$, a sign pattern $\varepsilon$ for $N$, and let $Z$ be the collection of pairs $(K, \chi)$ satisfying the hypotheses of the previous theorem. Then, for any fixed eigenform $\varphi \in S^{\varepsilon}(\mathcal{O})$, the twisted central values $L\left(\frac{1}{2}, \varphi_{K} \otimes \chi\right)$ for $(K, \chi) \in Z$ are either all zero or all nonzero.

Note that one can compute examples of $B$ such that $|Z|>1$, so that this corollary has some content, however we do not know if there are infintely many such $Z$.

Theorem 6.12. Suppose $\varphi \in S(\mathcal{O})$ is an eigenform such that $\varphi(1) \neq 0$. Then there exists a character $\chi$ of $\mathrm{Cl}(K)$ such that $L\left(\frac{1}{2}, \varphi_{K} \otimes \chi\right) \neq 0$.

Proof. By orthogonality of characters, we have $\sum_{\chi \in \widehat{\mathrm{Cl}}(K)} P_{K, \chi}(\varphi)=h_{K} \varphi(1)$. Hence some $P_{K, \chi}(\varphi) \neq 0$ if $\varphi(1)$ is.

When $h_{K}=1$, this theorem overlaps with the previous one.

Remark 6.13. The above argument similarly works (via a different linear combination of characters) if $\varphi$ is nonzero on some element in the image of $\mathrm{Cl}(K)$. This fact was used in [MV07] to make the same conclusion for any eigenform $\varphi$ if $D$ is sufficiently large (and in fact get a lower bound on how many $\chi$ satisfy $\left.L\left(\frac{1}{2}, \varphi_{K} \otimes \chi\right) \neq 0\right)$. Our point is that the nonvanishing of $\varphi$ at a certain value also implies the nonvanishing of twisted $L$-values for small $D$. 
We also remark that Proposition 6.2 implies $P_{K, \chi^{-1}}(\varphi)=P_{K, \chi}\left(\sigma_{N}(\varphi)\right)=\varepsilon_{N}(\varphi) P_{K, \chi}(\varphi)$. This corresponds to the fact that $L\left(\frac{1}{2}, \varphi_{K} \otimes \chi\right)=\varepsilon\left(\frac{1}{2}, \varphi_{K} \otimes \chi\right) L\left(\frac{1}{2}, \varphi_{K} \otimes \chi^{-1}\right)$, because the representations $\pi_{K} \otimes \chi$ and $\pi_{K} \otimes \chi^{-1}$ are contragredient. In particular, another statement one can make about non-vanishing of twists is that $L\left(\frac{1}{2}, \varphi_{K} \otimes \chi\right) \neq 0$ if and only if $L\left(\frac{1}{2}, \varphi_{K} \otimes \chi^{-1}\right) \neq 0$.

Example 6.14. We continue the example $N=154$ from Example 3.2 and the notation therein. Here the fields $K=\mathbb{Q}(\sqrt{-D})$ with class number 1 which embed in $B$ are those with $D \in\{4,11,67,163\}$. Since $\varphi_{1}, \varphi_{2}$ are zero-free, $L\left(\frac{1}{2}, \varphi_{K}\right)=L\left(\frac{1}{2}, \varphi\right) L\left(\frac{1}{2}, \varphi \otimes \eta_{D}\right) \neq 0$ for $\varphi=\varphi_{1}, \varphi_{2}$ and each such $K$. Since $\varphi_{5}$ has root number -1 , each $L\left(\frac{1}{2}, \varphi_{5, K}\right)=0$.

Now $\varphi_{3}$ and $\varphi_{4}$ correspond to weight 2 rational newforms in $S_{2}(154)$ with root number +1 . All elliptic curves of conductor 154 and root number +1 are rank 0 , so $L\left(\frac{1}{2}, \varphi_{3}\right)$ and $L\left(\frac{1}{2}, \varphi_{4}\right)$ are nonzero. Hence for $\varphi \in\left\{\varphi_{3}, \varphi_{4}\right\}$, we have $L\left(\frac{1}{2}, \varphi \otimes \eta_{D}\right) \neq 0$ if and only if $P_{K}(\varphi) \neq 0$. Recall that $\sigma_{2}$ fixes the ideal classes in $X_{3}$. Thus Proposition 6.5 implies $\mathbb{Z}[i]$ must embed into the left order $\mathcal{O}_{\ell}\left(x_{5}\right)=\mathcal{O}_{\ell}\left(x_{6}\right)$ associated to $X_{3}$. (Alternatively one can check in Magma that $\mathcal{O}_{\ell}\left(x_{5}\right)$ has 4 units and apply Remark 6.1.) Similarly, one sees that $\mathbb{Z}\left[\frac{1+\sqrt{-11}}{2}\right]$ embeds in the left order associated to $X_{1}$. Consequently, we see $L\left(\frac{1}{2}, \varphi_{3} \otimes \eta_{4}\right) \neq 0, L\left(\frac{1}{2}, \varphi_{3} \otimes \eta_{11}\right)=0, L\left(\frac{1}{2}, \varphi_{4} \otimes \eta_{4}\right)=0$, and $L\left(\frac{1}{2}, \varphi_{4} \otimes \eta_{11}\right) \neq 0$.

We find this example interesting because here it is trivial zeroes that force the elliptic curves associated to $\varphi_{3} \otimes \eta_{11}$ and $\varphi_{4} \otimes \eta_{4}$ to have rank at least 2. However, we expect that this phenomenon of trivial zeroes forcing analytic rank $\geq 2$ is quite rare-possibly it only happens finitely often.

\section{REFERENCES}

[BM13] Valentin Blomer and Philippe Michel, Hybrid bounds for automorphic forms on ellipsoids over number fields, J. Inst. Math. Jussieu 12 (2013), no. 4, 727-758. $\uparrow 4$

[BCP97] Wieb Bosma, John Cannon, and Catherine Playoust, The Magma algebra system. I. The user language, J. Symbolic Comput. 24 (1997), no. 3-4, 235-265. Computational algebra and number theory (London, 1993). $\uparrow 5$

[DV13] Lassina Dembélé and John Voight, Explicit methods for Hilbert modular forms, Elliptic curves, Hilbert modular forms and Galois deformations, Adv. Courses Math. CRM Barcelona, Birkhäuser/Springer, Basel, 2013, pp. 135-198. $\uparrow 2$

[DF87] Persi Diaconis and David Freedman, A dozen de Finetti-style results in search of a theory, Ann. Inst. H. Poincaré Probab. Statist. 23 (1987), no. 2, suppl., 397-423 (English, with French summary). $\uparrow 5.2$

[Gro87] Benedict H. Gross, Heights and the special values of L-series, Number theory (Montreal, Que., 1985), CMS Conf. Proc., vol. 7, Amer. Math. Soc., Providence, RI, 1987, pp. 115-187. ^2.1, 6.1

[GP91] Benedict H. Gross and Dipendra Prasad, Test vectors for linear forms, Math. Ann. 291 (1991), no. 2, 343-355. $\uparrow 6.2$

[HPS89] H. Hijikata, A. Pizer, and T. Shemanske, Orders in quaternion algebras, J. Reine Angew. Math. 394 (1989), 59-106. $\uparrow 2,2$

[ILS00] Henryk Iwaniec, Wenzhi Luo, and Peter Sarnak, Low lying zeros of families of L-functions, Inst. Hautes Études Sci. Publ. Math. 91 (2000), 55-131 (2001). ^1.3

[Jac87] Hervé Jacquet, Sur un résultat de Waldspurger. II, Compositio Math. 63 (1987), no. 3, 315-389 (French). $\uparrow 6.2$

[JC01] Hervé Jacquet and Nan Chen, Positivity of quadratic base change L-functions, Bull. Soc. Math. France 129 (2001), no. 1, 33-90 (English, with English and French summaries). ^1.1, 6.2 
[Mar17] Kimball Martin, The Jacquet-Langlands correspondence, Eisenstein congruences, and integral L-values in weight 2, Math. Res. Lett. 24 (2017), no. 6, 1775-1795. Corrected version available at http://arxiv.org/abs/1601.03284. $\uparrow 1.1,2,2$

[Mar18a] — Refined dimensions of cusp forms, and equidistribution and bias of signs, J. Number Theory 188 (2018), 1-17. $\uparrow 3.2,3.2,3.2,3.3,3.3,5.1,6.7$

[Mar18b] Congruences for modular forms mod 2 and quaternionic S-ideal classes, Canad. J. Math. 70 (2018), no. 5, 1076-1095. $11.2,3.1,3.9,3.11,4.3$

[Mara] _ The basis problem revisited. Trans. Amer. Math. Soc., to appear, http://arxiv.org/abs/arXiv:1804.04234. $\uparrow 2,2$

[Marb] An on-average Maeda-type conjecture in the level aspect. http://arxiv.org/abs/1912.06911. $\uparrow 1.1,1.2,1.3,5.1,5.2,5.4,5.2,5.2,5.4$

[Mic04] P. Michel, The subconvexity problem for Rankin-Selberg L-functions and equidistribution of Heegner points, Ann. of Math. (2) 160 (2004), no. 1, 185-236. ^1.3, 6.1

[MV07] Philippe Michel and Akshay Venkatesh, Heegner points and non-vanishing of Rankin/Selberg L-functions, Analytic number theory, Clay Math. Proc., vol. 7, Amer. Math. Soc., Providence, RI, 2007, pp. 169-183. 个1.1, 1.3, 6.1, 6.13

[Sage] The Sage Developers, Sagemath, the Sage Mathematics Software System (Version 8.9), 2019. https://www. sagemath.org. $\uparrow 5$

[Tun83] Jerrold B. Tunnell, Local $\epsilon$-factors and characters of GL(2), Amer. J. Math. 105 (1983), no. 6, 1277-1307. $\uparrow 6.2,6.2$

[Wal85] J.-L. Waldspurger, Sur les valeurs de certaines fonctions $L$ automorphes en leur centre de symétrie, Compositio Math. 54 (1985), no. 2, 173-242 (French). ^1.1, 1.3, 6.2

[Wei73] P. J. Weinberger, Exponents of the class groups of complex quadratic fields, Acta Arith. 22 (1973), 117-124. $\uparrow 1.3,6.7$

[Wie19] Jordan Wiebe, Arithmetic in Quaternion Algebras and Quaternionic Modular Forms, 2019. PhD Thesis, University of Oklahoma. $\uparrow 3.2$

E-mail address: kimball.martin@ou.edu

Department of Mathematics, University of Oklahoma, Norman, OK 73019 USA

E-mail address: jwiebe@ou.edu 